\title{
Intersections of quadrics, moment-angle manifolds and connected sums
}

\author{
SAMUEL GITLER \\ SANTIAgo LÓPEZ DE MEDRANo
}

For the intersections of real quadrics in $\mathbb{R}^{n}$ and in $\mathbb{C}^{n}$ associated to simple polytopes (also known as universal abelian covers and moment-angle manifolds, respectively) we obtain the following results:

(1) Every such manifold of dimension greater than or equal to 5, connected up to the middle dimension and with free homology, is diffeomorphic to a connected sum of sphere products. The same is true for the manifolds in infinite families stemming from each of them. This includes the moment-angle manifolds for which the result was conjectured by F Bosio and L Meersseman.

(2) The topological effect on the manifolds of cutting off vertices and edges from the polytope is described. Combined with the result in (1), this gives the same result for many more natural, infinite families.

(3) As a consequence of (2), the cohomology rings of the two manifolds associated to a polytope need not be isomorphic, contradicting published results about complements of arrangements.

(4) Auxiliary but general constructions and results in geometric topology.

14P25, 57R19; 57S25, 57R65

To Sylvain Cappell, on his $65^{\text {th }}$ birthday

\section{Introduction}

The geometric topology of the generic intersection of two homogeneous coaxial quadrics in $\mathbb{R}^{n}$ was studied by the second author in [26] where it was shown that its intersection with the unit sphere is in most cases diffeomorphic to a triple product of spheres or to the connected sum of sphere products. The proof involved a geometric description of the group actions on them and of their polytope quotients as well as a splitting of the homology groups of those manifolds. It also relied heavily on a normal form for them and many related computations. The part about group actions, polytopes and homology splitting was equally valid for the intersection of any number of such 
quadrics, but the obstacle to extending the main result for more than two turned out to be the hopeless-looking problem of finding their normal forms, close to that of classifying all simple polytopes.

The study of those intersections continued in other directions, especially those related to the projectivizations of their complex versions (known now as $L V-M$ manifolds; see the second author and Verjovsky [27], Meersseman and Verjovsky [28; 29; 30], Loeb and Nicolau [22] and Bosio and Meersseman [7; 8]), giving many new examples of nonalgebraic complex manifolds which fibre over toric varieties.

Following this line of research, in [8] a deep study of LV-M manifolds included also important advances in the study of the geometric topology of the intersection of $k$ homogeneous quadrics in their complex versions (the manifolds now called moment-angle manifolds). The main questions addressed were the following:

(1) Whether they can always be built up from spheres by repeatedly taking products or connected sums: they produced new examples for any $k$ which are so, but also showed how to construct many cases which are not. Many interesting questions arose, including a specific conjecture.

(2) The transition between different topological types when the generic condition is broken at some point of a deformation (wall-crossing).

(3) A product rule of their cohomology ring (in the spirit of the description of the homology of $Z$ given in [26]) and its applications to question (1).

Meanwhile, and independently, essentially the same manifolds were constructed in a more abstract way, where the main objective was to study the algebraic topology of some important quotients of them called initially toric manifolds and now quasitoric manifolds; see Davis and Januszkiewicz [14]. This article originated an important development through the work of many authors, and there is a vast and deep literature along those lines for which the reader is referred to Buchstaber and Panov [10]. Yet for a long time no interchange occurred between the two lines of research involving the same objects, until small connections appeared in the final version of [8] and in Denham and Suciu [15]. In particular, it turned out that examples relevant to question (1) above were known to these authors (see [6]), and in [5] Baskakov had a product rule for the cohomology ring, similar but dual to that of [8] mentioned in (3) above. All those examples were independent and more or less simultaneous, yet both product rules followed from an earlier computation by Buchstaber and Panov of the cohomology ring. ${ }^{1}$

\footnotetext{
${ }^{1}$ In the first version of [8] the product rule was derived from results in de Longueville [23], while in the published version it relied on [10].
} 
One recent expression of the line of research derived from [14] is the article by Bahri, Bendersky, Cohen and the first author [2] where a far-reaching generalization is made and a general geometric splitting formula is derived that is, in particular, a very good tool for understanding the relations among the homology groups of different spaces. This understanding turned out to be fundamental for us in tracing a way through the abstract situation of the intersection of $k>2$ quadrics, thus combining efficiently both approaches to the subject as expressed in $[26 ; 2]$ to obtain the results in the present article. Nevertheless, these results do not depend logically on those articles and are actually more geometric than any of them, involving practically no computations.

The results in this article follow the three paths outlined in [8] mentioned above, but including now all the intersections of quadrics and not only the moment-angle manifolds.

(1) In Section 1 we identify very general families of manifolds that are indeed diffeomorphic to connected sums of sphere products. The main result there is Theorem 1.3 (of which the following is a simplified version), where $Z$ denotes an intersection of quadrics and $Z^{J}$ is an infinite family obtained from $Z$ by increasing the dimension of its coordinate spaces.

Theorem 1.3 Assume $Z$ is of dimension $2 c$ and $(c-1)$-connected, where $c \geq 2$. Then any $Z^{J}$ of dimension at least 5 is diffeomorphic to a connected sum of sphere products.

Particular members of such families are those moment-angle manifolds for which the result answers the Bosio-Meersseman conjecture in [8]. The dimension hypothesis only excludes the simply connected 4-dimensional ones, for which the result should also be true but our methods only show that they are homeomorphic to a connected sum of copies of $S^{2} \times S^{2}$. A weaker result in the odd-dimensional case is given in Theorem 1.4, while Theorems 1.1 and 1.2 are relative results used in the proofs of the main theorems, but which have other applications. As a byproduct of the proofs, a simpler and neater proof of the result in [26] is also obtained.

(2) In Section 2 we give an explicit topological description of some of the transitions, mainly that of cutting off a vertex or an edge of the associated polytope (operations $Z_{\mathrm{v}}$ and $Z_{\mathrm{e}}$ ). We show that, under simple assumptions, these operations preserve connected sum of sphere products and adequately modified (operations $Z_{\mathrm{v}}^{\prime}$ and $Z_{\mathrm{e}}^{\prime}$ ) they can be combined with the theorems in Section 1 to give new infinite families that are diffeomorphic to connected sums of sphere products, the final result along this line being the following. 
Theorem 2.4 If $Z=Z(P)$ is a connected sum of sphere products and is simply connected of dimension at least 5, then any manifold obtained from $Z$ by repeatedly applying the $Z^{J}, Z_{v}^{\prime}$ and $Z_{e}^{\prime}$ constructions (any number of times each and in any order) is also a connected sum of sphere products.

The initial manifold can be any sphere, any highly connected manifold $Z(P)$ as in Theorems 1.3 and 1.4 or any simply connected one with $m-d=3$ of dimension at least 5 that is not a triple sphere product [26].

The first results in Section 2 include cases where the initial manifold is quite general and we make a similar conjecture specific for moment-angle manifolds. A proof of that conjecture under a restrictive hypothesis is enough for us to describe the topology of other important examples taken from [8]: the manifolds associated to the truncated cube.

(3) In Section 3 we use our knowledge of the above examples to give a proof that their cohomology rings are not isomorphic as ungraded rings, not even when taken with $\mathbb{Z}_{2}$ coefficients. Thus we show that the product rule for the moment-angle manifolds has to be drastically modified in the general situation. This contradicts a result by de Longueville in [23]. We only state the modified rule, and leave the details for another publication.

In Section 0 we recall the necessary definitions and known results and in Section 2.1 some elementary topological constructions are defined and explored. In the appendices we state and prove some results about specific differentiable manifolds, which are used in Sections 1 and 2.

In the process of solving these problems several new questions and conjectures have arisen. Some extensions of our results are immediate, others should be possible by continued work along the same lines. Others seem to need a whole new approach.

Acknowledgements This research was initiated by a discussion with Frédéric Bosio and Laurent Meersseman around the article [1] and continued through fruitful conversations with them and with Tony Bahri, Martin Bendersky, Fred Cohen, Vinicio Gómez Gutiérrez, Francisco González Acuña and Alberto Verjovsky.

Part of this research was done while the second author enjoyed the warm and stimulating hospitality of the NYU Courant Institute, for which he is most grateful to Sylvain Cappell. Invitations to Princeton University by William Browder and to the CUNY Graduate Center by Martin Bendersky and Maxim Laurentiu were also very useful and encouraging. He was also partially supported by DGAPA-UNAM grant PAPIITIN102009. 


\section{Preliminaries}

\subsection{Basic definitions}

Let $m>d>0$ be integers and $k=m-d-1$,

$$
\Lambda_{i} \in \mathbb{R}^{k}, i=1, \ldots, m, \quad \Lambda=\left(\Lambda_{1}, \ldots, \Lambda_{m}\right) .
$$

If $I \subset\{1, \ldots, m\}$ we will denote by $\Lambda_{I}$ the sequence of $\Lambda_{i}$ with $i \in I$.

We will assume the (generic) weak hyperbolicity condition:

If I has $k$ or less elements then the origin is not in the convex hull of $\Lambda_{I}$.

Let $Z=Z(\Lambda)$ be the submanifold ${ }^{2}$ of $\mathbb{R}^{m}$ given by the equations

$$
\sum_{i=1}^{m} \Lambda_{i} x_{i}^{2}=0, \quad \sum_{i=1}^{m} x_{i}^{2}=1 .
$$

It is immediate that this manifold is nonempty if, and only if, the origin of $\mathbb{R}^{k}$ is in the convex hull of the $\Lambda_{i}$.

The manifold $Z$ is smooth, since the weak hyperbolicity condition is equivalent to the regularity of the system of its defining equations. It is immediate that it is stably parallelizable. A simple construction (see Section 1) shows that it is always the boundary of a parallelizable manifold.

Let $Z^{\mathbb{C}}=Z^{\mathbb{C}}(\Lambda)$ be the manifold defined in $\mathbb{C}^{m}$ by the equations

$$
\sum_{i=1}^{m} \Lambda_{i}\left|z_{i}\right|^{2}=0, \quad \sum_{i=1}^{m}\left|z_{i}\right|^{2}=1 .
$$

Of course, $Z^{\mathbb{C}}$ is a particular (but very important) case of a $Z$ manifold if we write its equations in real coordinates in $\mathbb{R}^{2 m}$, each $\Lambda_{i} \in R^{k}$ appearing twice.

It was shown in [26] that for $k=2$, all manifolds $Z^{\mathbb{C}}$ and almost all simply connected manifolds $Z$ are either empty, a product of three spheres or a connected sum of

${ }^{2}$ These intersections of quadrics were first studied in Wall [33], Chaperon [12], Hirzebruch [20] and [26]. The manifolds $Z^{\mathbb{C}}$ were first studied in Camacho, Kuiper and Palis [11] and [12; 25] where they arose (and reappeared recently in Chaperon and second author's work [13]) in the context of dynamical systems. $Z, Z^{\mathbb{C}}$ are essentially the same as the manifolds constructed independently in [14], known as universal abelian covers of polytopes and moment-angle manifolds, respectively. $Z$ and the constructions $Z_{+}, Z^{\prime}, Z^{J}$ described below, can be expressed in the framework of the generalized moment-angle complexes defined in [2]. 
manifolds, each of which is a product of two spheres. We will call this type of sum connected sum of sphere products (implicitly implying that each summand is the product of only two spheres).

It is easy to see that the collections of manifolds $Z(\Lambda)$ and $Z^{\mathbb{C}}(\Lambda)$ are both closed under products so one gets examples for high $k$, where $Z$ and $Z^{\mathbb{C}}$ is a product of any number of spheres and any number of connected sums of sphere products, which have therefore free homology groups and easily described cohomology rings.

In [8] it was shown that for $k \geq 3$ the manifold $Z^{\mathbb{C}}$ may be considerably more complicated (see also [6]) and that for $k \geq 5$ its homology may have a lot of torsion. But they also conjectured that under certain conditions one obtains again the same kind of connected sums. The results of this article imply that this Bosio-Meersseman conjecture is true and we obtain the same conclusion in many more cases.

\subsection{Group actions and polytopes}

The manifold $Z$ admits a $\mathbb{Z}_{2}^{m}$ action by changing the signs of the coordinates. The quotient is a simple polytope $P$ which can be identified with the intersection of $Z$ and the first orthant of $\mathbb{R}^{m}$. It follows that $Z$ can be reconstructed from this intersection by reflecting it on all the coordinate hyperplanes.

By a simple change of coordinates $r_{i}=x_{i}^{2}$, this quotient can be identified with the $d$-dimensional convex polytope $P$ given by

$$
\sum_{i=1}^{m} \Lambda_{i} r_{i}=0, \quad \sum_{i=1}^{m} r_{i}=1, \quad r_{i} \geq 0
$$

The weak hyperbolicity condition is equivalent to the fact that $P$ is a simple polytope, meaning that each vertex is exactly in $d$ facets of $P$.

The facets of $P$ (ie, its $(d-1)$-dimensional faces) are the nonempty intersections of $P$ with the coordinate hyperplanes $\left\{r_{i}=0\right\}$. It follows that a nonempty $Z$ is connected if, and only if, all these intersections are nonempty, or equivalently, if $P$ has exactly $m$ facets, since otherwise different components of $P$ would lie in different sides of an hyperplane $\left\{x_{i}=0\right\}$. In that case $Z=Z_{*} \times \mathbb{Z}_{2}^{h}$, where $Z_{*}$ is a connected component of $Z$ and $h$ is the number of those coordinate hyperplanes not touching $Z$.

If we introduce a new facet by intersecting $P$ with a half space $H=\left\{\sum_{i=1}^{m} a_{i} r_{i} \geq a\right\}$ whose boundary does not contain any vertex of $P$ we obtain a new simple polytope $P_{H}$. By introducing a new coordinate $r_{0}=\sum_{i=1}^{m} a_{i} r_{i}-a$ we can put $P_{H}$ in $\mathbb{R}^{m+1}$ with $r_{0} \geq 0$. By simple manipulations of the equations of $P_{H}$ and changes of coordinates 
of the type $r_{i}^{\prime}=b_{i} r_{i}, i=0, \ldots, m$ we get a system of equations for $P_{H}$ of the same form as that of $P$.

This implies, by induction on the number of facets, that any simple polytope can be expressed in the above form ${ }^{3}$ and is therefore the quotient polytope of a connected manifold $Z$. We will denote it by $Z(P)$, since it can be recovered combinatorially 4 from $P$ as a well-determined quotient of $P \times \mathbb{Z}_{2}^{m}$, where $m$ is the number of facets of $P$.

This means that the connected $Z$ coincide with the manifolds constructed in [14], called by them universal abelian covers.

It is known that $Z(P)$ is $(c-1)$-connected if, and only if, the intersection of any $c$ facets of $P$ is nonempty. ${ }^{5}$ Following [8], such a $P$ is called dual $c-n e i g h b o r l y$ and simply dual neighborly if it is $[d / 2]$-dual neighborly. The Bosio-Meersseman conjecture says that if $P$ is even-dimensional and dual-neighborly then $Z^{\mathbb{C}}$ is a connected sum of sphere products.

The manifold $Z^{\mathbb{C}}$ admits an action of the $m$-torus $T^{m}=\left(S^{1}\right)^{m}$ by multiplication of unit complex numbers on each of the coordinates. The quotient by this action is the same simple polytope $P$ and $Z^{\mathbb{C}}$ can be reconstructed from $P \times T^{m}$ by making the obvious identifications on the facets of $P$. The orbits of this action over a facet $F$ correspond to the points where some coordinate $z_{i}$ vanishes and are thus the points where the corresponding factor of the torus acts trivially.

If $P$ has exactly $m$ facets, any orbit of the action of $T^{m}$ on $Z^{\mathbb{C}}$ is nullhomotopic in $Z^{\mathbb{C}}$. This is because it can always be deformed to an orbit over a facet of $P$, where one of the factors of $T^{m}$ acts trivially and so the orbit collapses into an orbit of a $T^{m-1}$-action. This new orbit can be deformed to a $T^{m-1}$ orbit over another facet of $P$ where another factor acts trivially. Since any factor of $T^{m}$ acts trivially on some facet we end up with a deformation of the original orbit into a point.

\footnotetext{
${ }^{3}$ This Realization Theorem is alluded to in [33] as a general correspondence known as the Gale diagram of the polytope. The perfect correspondence between polytopes and the manifolds $Z$ is mentioned in [26], but its precise statement and proof were not included after the author learned about the Gale diagram from Wall's paper. A detailed proof using the Gale Transform is given in [8, Theorem 0.14]. The simple proof by induction on the number of facets of $P$ is due to Vinicio Gómez Gutiérrez.

${ }^{4}$ One can deduce from [8] that combinatorially equivalent polytopes are in fact diffeomorphic as manifolds with corners, and thus there is really only one diffeomorphism type $Z(P)$. We will not be using this rigidity result since all our constructions can be given in terms of the equations and the uniqueness follows in general from the results themselves.

${ }^{5}$ The homology groups of $Z$ follow from the computations given in [26] or [2]. An argument for the fundamental group is given in [26].
} 
This proves that in that case $Z^{\mathbb{C}}$ is simply connected, since any loop in it can be moved away from the manifolds lying over the facets of $P$ (which have codimension 2) and so lies in the open set interior $(P) \times T^{m}$ and can therefore be deformed into a single orbit which in turn deforms into a point. From the same homology computations as above, $Z^{\mathbb{C}}$ is then $2 c$-connected if, and only if, $P$ is dual $c$-neighborly, and therefore always actually $2-$ connected.

On the contrary, if $P$ has $m-h$ facets then $Z^{\mathbb{C}}=Z_{*}^{\mathbb{C}} \times \boldsymbol{T}^{h}$ (for a proof of this, see [8, Lemma 0.10]). In that case the orbits of $T^{m}$ are not all nullhomotopic and $Z^{\mathbb{C}}$ is not simply connected.

The 2-connected $Z^{\mathbb{C}}$ coincide with the manifolds constructed in [14], called now moment-angle manifolds. This fact follows from the realization of a polytope as the quotient of a connected manifold $Z$ (see footnote 3 ).

We can therefore refer to the manifold $Z^{\mathbb{C}}(P)$, by which we mean the diffeomorphism type of any 2 -connected manifold $Z^{\mathbb{C}}(\Lambda)$ whose quotient polytope is $P$. It was shown in [8] that this diffeomorphism type and the $T^{m}$ action are determined by the combinatorial type of $P$.

\section{Opening books}

Let $\Lambda^{\prime}$ be obtained from $\Lambda$ by adding an extra $\Lambda_{1}$ (so $\Lambda^{\prime}$ satisfies also weak hyperbolicity) which we interpret as the coefficient of a new variable $x_{0}$, so we get the manifold $Z^{\prime}$ :

$$
\begin{gathered}
\Lambda_{1}\left(x_{0}^{2}+x_{1}^{2}\right)+\sum_{i>1} \Lambda_{i} x_{i}^{2}=0, \\
x_{0}^{2}+x_{1}^{2}+\sum_{i>1} x_{i}^{2}=1 .
\end{gathered}
$$

Let $Z_{+}$be the intersection of $Z$ with the half space $x_{1} \geq 0$ and $Z_{+}^{\prime}$ the intersection of $Z^{\prime}$ with the half space $x_{0} \geq 0$. The boundary of $Z_{+}^{\prime}$ is $Z$. This shows that $Z$ is always the boundary of a parallelizable manifold.

$Z_{+}$admits an action of $\mathbb{Z}_{2}^{m-1}$ by changing signs on all the variables except $x_{1}$ and the quotient is again $P$. In other words, $Z_{+}$can be obtained from $P \times \mathbb{Z}_{2}^{m-1}$ by making all the identifications on the facets of $P$ except for the one corresponding to $x_{1}=0$ (or, equivalently, by reflecting $P$ on all the coordinate hyperplanes except $x_{1}=0$ ).

Consider also the manifold $Z_{0}$ which is the intersection of $Z$ with the subspace $x_{1}=0$. $Z_{0}$ is the boundary of $Z_{+}$. So

$$
Z_{0} \subset Z_{+} \subset Z \subset Z_{+}^{\prime} \subset Z^{\prime}
$$


For example, $Z_{+}\left(\Delta^{n}\right)=D^{n}$ that can be obtained from $\Delta^{n} \times \mathbb{Z}_{2}^{n}$ by making the identifications on $n$ of the faces of $\Delta^{n}$ (or, equivalently, by reflecting the spherical simplex $S^{n} \cap \mathbb{R}_{+}^{n+1}$ on all the coordinate hyperplanes but one). Also $Z_{+}\left(\Delta^{n} \times I\right)$ can be either $D^{n} \times S^{1}$ if we do not reflect it on a facet $\Delta^{n-1} \times I$ or $S^{n} \times D^{1}$ if we do not reflect it on a facet $\Delta^{n} \times p t$.

The quotient polytope $P^{\prime}$ of $Z^{\prime}$ is obtained from $P \times I$ by collapsing $P_{0} \times I$ into $P_{0}$, where the latter is the facet of $P$ corresponding to $x_{1}=0$ (that is, $P_{0}$ is the quotient polytope of $Z_{0}$ ). Following [28; 8] we call this construction the $b o o k^{6}$ on $P$.

Now, $S^{1}$ acts on $Z^{\prime}$ (rotating the coordinates $\left(x_{0}, x_{1}\right)$ ) with fixed set $Z_{0}$. Its quotient can be identified with $Z_{+}$. The map

$$
\left(x_{0}, x_{1}, x_{2}, \ldots, x_{m}\right) \mapsto\left(\sqrt{x_{0}^{2}+x_{1}^{2}}, x_{2}, \ldots, x_{m}\right)
$$

is a retraction from $Z^{\prime}$ to $Z_{+}$which restricts to the retraction from $Z$ to $Z_{+}$:

$$
\left(x_{1}, x_{2}, \ldots, x_{m}\right) \mapsto\left(\left|x_{1}\right|, x_{2}, \ldots, x_{m}\right) .
$$

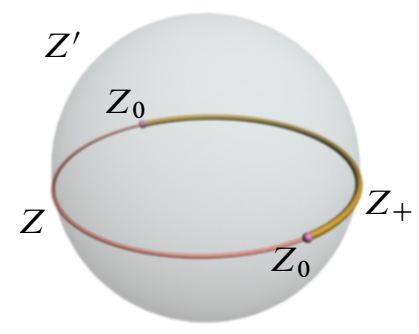

Observe further that this retraction restricted to $Z_{+}^{\prime}$ is homotopic to the identity: the homotopy preserves the coordinates $x_{i}, i \geq 2$ and folds gradually the half space $x_{0} \geq 0$ of the $x_{0}, x_{1}$ plane into the ray $x_{0}=0, x_{1} \geq 0$ preserving the distance to the origin.

So $Z$ is the double of $Z_{+}$and $Z^{\prime}$ is the double of $Z_{+}^{\prime}$ and $Z^{\prime}$ is the open book with page $Z_{+}$and trivial holonomy. ${ }^{7}$

The inclusion of $Z=\partial Z_{+}^{\prime}$ into $Z_{+}^{\prime}$ is homotopic to the retraction from $Z$ to $Z_{+}$.

\footnotetext{
${ }^{6}$ This construction is implicit in [26] and was much used in the process of proving its main Theorem 2. The picture of the pentagonal book appears already in [33, page 413]. In some literature stemming from [14] it is known as the Buchstaber construction and some polytope theorists call it the wedge on $P$.

${ }^{7}$ In the notation of Section $2.1, Z=\mathcal{D}\left(Z_{+}\right), Z^{\prime}=\mathcal{D}\left(Z_{+}^{\prime}\right)$ and $Z^{\prime}=\mathcal{T} \mathcal{O B}\left(Z_{+}\right)$.
} 


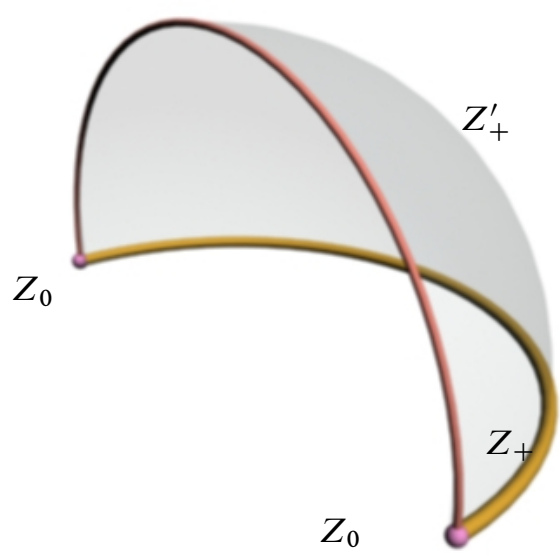

The construction of $Z^{\prime}$ from $Z$ can be generalized as follows: Let $J=\left(j_{1}, \ldots, j_{m}\right)$ be a vector of positive integers and $|J|=\sum j_{i}$. Then we construct a new configuration $\Lambda^{J}$ obtained from $\Lambda$ by repeating $j_{i}$ times the coefficient $\Lambda_{i}$ for each $i$ and thus a new corresponding manifold $Z^{J}$. The manifold $Z^{J}$ has a natural $O\left(j_{1}\right) \times \cdots \times O\left(j_{m}\right)$ action whose quotient is the same simple polytope $P$, as well as its own $\mathbb{Z}_{2}^{|J|}$ action with quotient a polytope $P^{J}$.

For example, if $J=(2,1, \ldots, 1)$ we have $Z^{J}=Z^{\prime}$. If $j$ is a positive integer we denote by $\underline{j}$ the vector $(j, j, \ldots, j)$. Thus $Z^{\underline{1}}=Z$ and $Z^{\underline{2}}=Z^{\mathbb{C}}$.

Independently of any construction related to quadrics, the $Z^{J}$ construction appeared in Bahri, Bendersky, Cohen and the first author [4] (already in its first draft of 2008) in the context of toric manifolds and generalized moment-angle complexes. There it is shown how to describe explicitly the polytope $P^{J}$ and how to construct a new toric manifold $M^{J}$ associated to $P^{J}$ from a toric manifold $M$ associated to $P$.

In fact, all our constructions can be expressed within the context of the generalized moment-angle complexes of [2] (but not inside any of the many previous generalizations of moment-angle manifolds) and the general splitting formula proved there provides an excellent geometric tool for understanding (among other things) the relations among the homology groups of all these manifolds. It is out of this understanding that were discovered the geometric relations used in the following proofs.

We will prove that certain families of manifolds $Z$ are connected sums of sphere products. The rough idea of the proof in each case is as follows.

(i) We show that the lower-dimensional manifolds in the family, being highly connected, are connected sums of sphere products. 
(ii) Then we use the geometric relation between the manifolds $Z$ and $Z^{\prime}$ to prove the induction step that allows us to cover all the high-dimensional ones.

Part (i) can be derived from the classification results about highly connected manifolds (see, among others, Kervaire and Milnor [21] and Wall [31; 32]). This type of result usually involves practically all the techniques of simply connected surgery (see Browder [9] and [21]) which include the number-theoretic and algebraic analysis of the middle-dimensional quadratic forms in order to represent homology classes by embedded spheres with trivial normal bundle, the construction and simplification of an adequate cobordism and the use of the $h$-cobordism theorem.

In our case we will not use those results but will use instead a variation of the arguments used in the induction step (ii) to prove (i) directly using the simple nature of our manifolds for which those constructions are elementary.

Part (ii) follows the spirit of those classification theorems, but is considerably simpler. We use the obvious fact that if $X$ is a simply connected sum of sphere products, then a basis of each homology group below the top dimension can be represented by embedded spheres with trivial normal bundle. A simple geometric lemma lets us represent various combinations of them by embedded spheres with trivial normal bundle inside $X \times I$ and disjoint from each other.

Then we use the natural cobordism $Z_{+}^{\prime}$ which has the crucial but simple property that all its homology comes from its boundary $Z$. Combining this with the previous fact we can use Theorem A1.1 in the Appendix to show that $Z_{+}^{\prime}$ is a connected sum along the boundary of trivial bundles over spheres, so the induction step follows easily for its double $Z^{\prime}$.

We start by the induction step (Theorem 1.1) and its variation that allows us to simplify the starting point of the induction (Theorem 1.2). These are interesting by themselves since they can be used in other situations. Then we will prove our two main results (Theorems 1.3 and 1.4).

Theorem 1.1 Assume $Z$ is simply connected and of dimension $d \geq 5$. If $Z$ is a connected sum of sphere products then $Z^{\prime}$ is also a connected sum of sphere products.

Proof We start with a lemma.

Lemma 1 If $X$ is a manifold of dimension $d$ and $\alpha_{1}, \alpha_{2} \in H_{i}(X)$ can be represented by disjoint embedded spheres $S_{1}, S_{2}$ with trivial normal bundle, then if $i \leq d-2$, $\alpha_{1}+\alpha_{2}$ can be represented by an embedded sphere with trivial normal bundle and disjoint from $S_{1}$ and $S_{2}$. 
Proof of the lemma Taking disjoint tubular neighborhoods of $S_{1}, S_{2}$ and joining them with a thin tube, we obtain a new manifold $X^{\prime}$ diffeomorphic to the connected sum along the boundary of two copies of $S^{i} \times D^{d-i}$. It is enough to prove the lemma for $X^{\prime}$. We can take for $X^{\prime}$ a standard model constructed as follows: take $S_{1}^{\prime}, S_{2}^{\prime}$ to be round spheres in $\mathbb{R}^{i+1} \subset \mathbb{R}^{d}$ of radius $1 / 2$ centered in $(1,0, \ldots, 0)$ and $(-1,0, \ldots, 0)$ together with the straight line segment $\sigma$ joining $(-1 / 2,0, \ldots, 0)$ and $(1 / 2,0, \ldots, 0)$. Now consider an $\epsilon$ neighborhood of this configuration in $\mathbb{R}^{d}$.

One can assume that the spheres with their standard orientations in $\mathbb{R}^{i+1}$ correspond to the classes $\alpha_{1}, \alpha_{2} \in H_{i}(X)$ : Because if $S_{i}^{\prime}$ corresponded to $-\alpha_{i}$, one can reflect the product $S^{i} \times D^{d-i}$ on the hyperplane $x_{2}=0$ of $\mathbb{R}^{d}$ and twist the tube around $\sigma$ accordingly. ${ }^{8}$

Taking the intersection of this neighborhood with $\mathbb{R}^{i+1}$ we obtain a manifold whose boundary is formed by three embedded spheres with trivial normal bundle in $\mathbb{R}^{i+1}$ and therefore also in $\mathbb{R}^{d}$. The outside sphere (adequately oriented) represents the sum $\alpha_{1}+\alpha_{2}$ and is disjoint from $S_{1}$ and $S_{2}$.

To apply the lemma to the situation of the theorem, observe that, since $Z$ is a connected sum of sphere products, all its homology groups are free and all the generators of the homology below the top dimension are represented by embedded spheres with trivial normal bundle. Furthermore, they can all be made disjoint inside $Z \times I$ by displacing them to different levels of the coordinate $t \in I$. They all have codimension at least 2 in $Z$ since $Z$ is simply connected, so they have codimension at least 3 in $Z \times I$. The lemma implies that not only the generators, but all elements of the homology below the top dimension can be represented by embedded spheres in $Z \times I$ with trivial normal bundle $^{9}$ where any finite collection of them can be represented by mutually disjoint ones.

As we showed earlier in this section, the inclusion of $Z=\partial Z_{+}^{\prime}$ into $Z_{+}^{\prime}$ is homotopic to the retraction $\rho: Z \rightarrow Z_{+}$.

Now, $\rho_{*}: H_{i}(Z) \rightarrow H_{i}\left(Z_{+}\right)$satisfies $\rho_{*} \iota_{*}=i d$, where $\iota_{*}$ is induced by the inclusion. So $\rho_{*}$ is an epimorphism and $H_{i}\left(Z_{+}\right)$can be identified with a subgroup of $H_{i}(Z)$ and

\footnotetext{
${ }^{8}$ When $i=d-1$ this last step cannot be achieved. For example, in $X=S^{d-1} \times S^{1}$ the homology class $\alpha+\alpha$ (for $\alpha$ the generator of $H_{d-1}(X)$ ) is not representable by an embedded sphere even if we can find two disjoint spheres each representing $\alpha$.

${ }^{9}$ This may not be true inside $Z$ itself: in $S^{2 c} \times S^{2 c}$ the sum of the generators can be represented by the diagonal sphere, but its normal bundle is the tangent bundle of $S^{2 c}$ which not trivial. No other embedded sphere representing that sum can have trivial normal bundle since its self-intersection number is 2 .
} 
is therefore free. The same argument applied to the fundamental groups shows $\pi_{1}\left(Z_{+}\right)$ is trivial.

The map in homology induced by the inclusion $Z \subset \partial Z_{+}^{\prime}$ has the same properties as $\rho_{*}$. It follows that all the homology of $Z_{+}^{\prime}$ comes from its boundary $Z$, is free in all dimensions ${ }^{10}$ and trivial in dimensions $d-1$ and $d$. Also, that $Z_{+}^{\prime}$ is simply connected.

So all the hypotheses of Theorem A1.1 in the Appendix are fulfilled and we conclude that $Z_{+}^{\prime}$ is the connected sum along the boundary of products of the form $S^{p} \times D^{d+1-p}$

Finally, $Z^{\prime}$, being the double of $Z_{+}^{\prime}$, is the connected sum of manifolds of the form $S^{p} \times S^{d+1-p}$.

Observe that all we have used about $Z$ is that it is simply connected, all its homology groups are free and every homology class below the top dimension can be represented by an embedded sphere inside $Z \times I$ with trivial normal bundle. Under these assumptions the same proof shows that $Z_{+}^{\prime}$ is the connected sum along the boundary of products of spheres with disks and that $Z$, being its boundary, is in fact a connected sum of sphere products. So, including the full induction on the size of $J$, we have actually proved more.

Theorem 1.2 Assume that $Z$ is simply connected and of dimension $d \geq 5$ and that all its homology groups are free and every homology class below the top dimension can be represented by an embedded sphere inside $Z \times I$ with trivial normal bundle. Then $Z$ is a connected sum of sphere products and so is $Z^{J}$ for any $J$.

As a first application, assume $Z$ is of dimension $2 c$ and $(c-1)$-connected (which means that the corresponding polytope is dual neighborly; see Section 0.2). If $c \geq 3$ then $Z$ clearly satisfies the hypotheses of Theorem 1.2 and every $Z^{J}$ is a connected sum of sphere products. For $c=2$ we only get that $Z$ is $h$-cobordant to a sum of copies of $S^{2} \times S^{2}$. But we can apply the construction in the proof of the theorems one more step up: one obtains that $Z^{\prime}$ is the boundary of a manifold $Z_{+}^{\prime \prime}$ which deforms down to $Z_{+}^{\prime}$. In $Z_{+}^{\prime}$ all the homology classes are in dimension 2 and can be represented by embedded spheres with trivial normal bundle, so the same is true for $Z_{+}^{\prime \prime}$.

\footnotetext{
${ }^{10}$ This was proved for $k=2$ in [26] using an explicit computation of the homology groups involved in that special case. A first proof in the general case used the stable splitting in [2] and the relationship between the polytopes $P$ and $P^{\prime}$, but its generality suggested the search for a simpler geometrical argument.
} 
Now the dimension of $Z_{+}^{\prime \prime}$ is 6 and Theorem A1.1 shows again that it is a connected sum along the boundary of copies of $S^{2} \times D^{4}$ and so its boundary $Z^{\prime}$ is a connected sum along the boundary of copies of $S^{2} \times S^{3}$. Applying now Theorem 1.1 inductively we obtain that all higher $Z^{J}$ are connected sums of products of spheres. So we have the following.

\section{Theorem 1.3 Assume $Z$ is of dimension $2 c$ and $(c-1)$-connected. Then}

(1) if $c \geq 3, Z$ and any $Z^{J}$ are connected sums of sphere products;

(2) if $c=2$, any $Z^{J}$ of dimension at least 5 is a connected sum of sphere products.

Remarks (1) The Bosio-Meersseman conjecture in [8, page 115], can be rephrased as follows: if $Z$ is of dimension $2 c$ and $(c-1)$-connected then $Z^{\mathbb{C}}$ is connected sums of sphere products, so we have proved in particular their conjecture for $c \geq 2 .^{11}$

(2) We conjecture now that if $Z$ is of dimension $2 c$ and $(c-1)$-connected then $Z^{J}$ is a connected sums of sphere products, that is, that Theorem 1.3 is true without any restrictions on $c$ and $J$. This is true for $c \geq 3$ and for $c=2$ the only missing case is $Z$ itself, where the topological classification of simply connected 4 manifolds shows only that $Z$ is homeomorphic to a connected sum of copies of $S^{2} \times S^{2}$. For $c=1$ we know that the conjecture is true for $Z$ (being a connected surface), for $Z^{J}$ when $J=\left(j_{1}, 1, \ldots, 1\right)$ (because we know $Z_{+}$) and for other restricted cases. For $J$ at least $\underline{2}$ it would follow from our conjecture preceding Theorem 2.2. This would also follow from [8, Theorem 6.3], but the proof of this result is not given and it is not clear to us how such a proof would avoid the problems we find in ours. Also, the result from McGavran [24] alluded to in [8] is stated only in the PL-category.

(3) Our result provides also a new, neater proof of the result in [26], starting from the basic $2 k$-dimensional $(k-1)$-connected manifolds when $k>2$ and with the simply connected 5-dimensional ones when $k=2$, all of which can be covered by the theorems of this section.

In the odd-dimensional case we have for the moment only weaker results: If $Z$ is of dimension $2 c+1$ and $(c-1)$-connected for $c>1$ we need to assume that all its homology groups are free, a condition that is automatic in the even-dimensional case. Nevertheless, it should be mentioned that the example of a manifold $Z$ with torsion

${ }^{11}$ Thus the counterexample to this conjecture announced in [1] is no such. To see directly that the argument there is not correct one can use the epimorphism part of the Hurewicz Theorem to check that $\pi_{6}(Z)$ is not as stated. 
in [8] does not have the high connectivity assumed here, so that hypothesis might still be redundant.

On the other hand we have a slightly weaker conclusion because we cannot exclude the possibility of having a connected sum of sphere bundles over spheres which are stably trivial over the embedded $(c+1)$-spheres but not trivial. When $c$ is odd the total space of those bundles has torsion so this possibility is excluded by our hypothesis. When $c$ is even the only possibility is the unit tangent bundle of $S^{c+1}$ which is trivial only for $c=2,6$. In this case they cannot be discarded by their torsion. Yet it is hard to see how those nontrivial bundles could have the high $\mathbb{Z}_{2}^{m}$ symmetry of our manifolds.

In any case this problem disappears for the manifold $Z^{\prime}$ by going up to the manifold $Z_{+}^{\prime \prime}$ as above. ${ }^{12}$

Theorem 1.4 Assume $Z$ is of dimension $2 c+1$ and $(c-1)$-connected where $c \geq 2$ and assume further that all the homology groups of $Z$ are free. Then

(i) $Z$ is a connected sum of stably trivial sphere bundles over spheres and if $J \neq 1$ then $Z^{J}$ is a connected sum of sphere products;

(ii) if $c$ is odd or if $c=2,6$, then $Z$ is a connected sum of sphere products.

The case $J=\underline{2}$ is a weak version of the Bosio-Meersseman conjecture in the odddimensional case.

Theorem 1.4 is definitely not true for $c=1$ : for the cube, $Z=S^{1} \times S^{1} \times S^{1}$.

In Theorems 1.3 and 1.4 the actual dimensions of the spheres can be deduced from the computation of the homology groups of the manifolds. These can be deduced from the combinatorics of the polytope as in $[26 ; 10 ; 8]$, or by using the stable splitting proved in [2]. Observe that we have also proved that the halved manifolds $Z_{+}^{\prime}$ are connected sums along the boundary.

\section{Cutting corners and making ends meet}

We study now the effect on the manifold $Z$ of cutting off parts of the polytope $P$ of dimension $d$. The general idea can be seen in the following situation.

Cutting off a vertex of $P$ gives a new polytope $P_{\mathrm{v}}$ and its corresponding manifold $Z_{\mathrm{v}}$. In $Z$ this has the effect of removing a number of open balls thus obtaining a manifold $Z_{\mathrm{v}+}$ with boundary. To obtain the manifold $Z_{\mathrm{v}}$ we have to take the double

12 Otherwise, remark that the double of a connected sum along the boundary of stably trivial $D^{c+1}-$ bundles over $S^{c+1}$ is a connected sum of trivial $S^{c+1}$-bundles over $S^{c+1}$. 


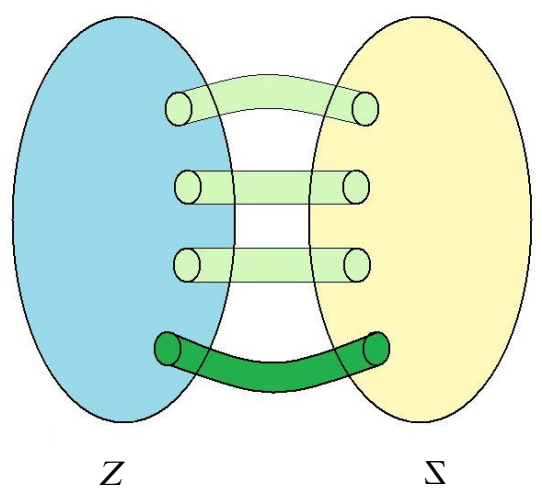

of $Z_{\mathrm{v}+}$ by taking two copies of it and identifying their boundaries (consisting each of a number of spheres). In the figure we have simulated this by joining those spheres by tubes $D^{1} \times S^{d-1}$.

We obtain the same result by taking two copies of $Z$ and performing several times the operation of removing a disk from each copy and joining their boundary spheres by a tube. Making the operation the first time amounts to taking the connected sum of the manifolds (imagine only the lower two disks and the darker tube). Since the resulting manifold is connected, each new time we perform the operation amounts to attaching a handle or, equivalently, taking the connected sum with $S^{1} \times S^{d-1}$. Thus the final result is the connected sum of two copies of $Z$ and a number of copies of $S^{1} \times S^{d-1}$ (one less than the number of open balls removed from $Z$ ).

To make precise this construction and some of its variants we introduce now some simple topological constructions and explore the relations among them.

\subsection{Doubles, open books and connected sums}

We work in the smooth category. In what follows we consider many manifolds with corners and we will use implicitly and systematically the fact that they have natural smoothings.

If $Q$ is a manifold with boundary, we denote its boundary by $\partial Q$ as usual.

Let $\mathcal{D} Q$ be the double of $Q$ :

$$
\mathcal{D} Q=\partial\left(Q \times D^{1}\right)
$$


Let $\mathcal{T O B}(Q)$ be the open book with trivial holonomy with leaf $Q$ and binding $\partial Q$, that is

$$
\mathcal{T O B}(Q)=Q \times S^{1} \cup \partial Q \times D^{2}=\partial\left(Q \times D^{2}\right) .
$$

If $M$ is a manifold without boundary and $M_{-1}$ is $M$ minus an open ball, $\mathcal{D} M_{-1}$ is diffeomorphic to $M \#(-M)$. In all cases we will consider below, $M$ admits an orientation reversing diffeomorphism, so $\mathcal{D} M_{-1}$ will be diffeomorphic to $M \# M$.

Let $\mathcal{G} M=\partial\left(M_{-1} \times D^{2}\right){ }^{13}$

So

$$
\mathcal{G} M=\mathcal{T} \mathcal{O B}\left(M_{-1}\right)
$$

As a simple example,

$$
\mathcal{G}\left(S^{n}\right)=S^{n+1} \text {. }
$$

If $Q, Q^{\prime}$ are connected $d$-manifolds, then we will consider their connected sum $Q \# Q^{\prime}$ (connecting the interiors of them). If $Q, Q^{\prime}$ have also connected nonempty boundaries we can take their connected sum along the boundary $Q \amalg Q^{\prime}$.

We explore now the relations between all these operations.

Clearly, if $M$ and $N$ are closed and connected and $Q, Q^{\prime}$ are connected with connected boundary then

(a) $(M \# N)_{-1}=M_{-1} \amalg N_{-1}$;

(b) $\partial\left(Q \amalg Q^{\prime}\right)=\partial Q \# \partial Q^{\prime}$;

(c) $\left(Q \amalg Q^{\prime}\right) \times D^{1}=\left(Q \times D^{1}\right) \amalg\left(Q^{\prime} \times D^{1}\right)$;

(d) $\mathcal{D}\left(Q \amalg Q^{\prime}\right)=\mathcal{D} Q \# \mathcal{D} Q^{\prime}$;

(e) $M \# Q=M_{-1} \amalg Q$.

Only part (e) seems to require an argument: Let $S$ be the sphere where $M_{-1}$ and $Q_{-1}$ are glued to form $M \# Q$ and $S \times D^{1}$ a tubular neighborhood of $S=S \times\{0\}$ in $M \# Q$. Take a small disk $D$ in $M \# Q$ around a point in $S \times\{1 / 2\}$ that intersects $S \times\{1 / 2\}$ in a disk. Then join $D$ to $\partial Q$ by a thin tube $T$ that does not touch $S \times\{1 / 2\}$. Then the manifold $R$ which is obtained from $M \# Q$ by removing the interior of $D \cup T$ is clearly diffeomorphic to $M \# Q$. On the other hand, cutting $R$ along the part of $S \times\{1 / 2\}$ not in $D$ one obtains on one side $M_{-1}$ and on the other a manifold diffeomorphic to $Q$. Joining them back one gets that $R$ is also the union of $M_{-1}$ and $Q$ joined along a disk in each of its boundaries, ie, $M_{-1} \amalg Q$ and (e) is proved.

More surprising are the following facts.

${ }^{13} \mathcal{G}$ stands for gyration and also for González Acuña (Fico) who defined and studied this operation in [17]. 
Lemma 2 (Fico's Lemmata, [17] ${ }^{14}$ )

(1) If $M$ and $N$ are connected, then we have $\mathcal{G}(M \# N)=\mathcal{G} M \# \mathcal{G} N$.

(2) $\mathcal{G}\left(S^{p} \times S^{q}\right)=\left(S^{p+1} \times S^{q}\right) \#\left(S^{p} \times S^{q+1}\right)$.

Proof For (1), just observe from (a) and (c) above that

$$
(M \# N)_{-1} \times D^{2}=\left(M_{-1} \amalg N_{-1}\right) \times D^{2}=\left(M_{-1} \times D^{2}\right) \amalg\left(N_{-1} \times D^{2}\right)
$$

and take the boundary using (b).

As for (2), observe that

$$
\left(S^{p} \times S^{q}\right)_{-1}=\left(S^{p} \times D^{q}\right) \cup_{D^{p} \times D^{q}}\left(D^{p} \times S^{q}\right)
$$

(the plumbing of two trivial disk bundles). Since its boundary is a sphere, this is not the connected sum $\left(S^{p} \times D^{q}\right) \amalg\left(D^{p} \times S^{q}\right)$, the underlying fact being that the two core spheres cannot be separated. However, it is easy to see, by separating the spheres in the product with $D^{1}$, that

$$
\left(\left(S^{p} \times D^{q}\right) \cup_{D^{p} \times D^{q}}\left(D^{p} \times S^{q}\right)\right) \times D^{1}=\left(S^{p} \times D^{q+1}\right) \amalg\left(D^{p+1} \times S^{q}\right) .
$$

Taking again the product with $D^{1}$, property (c) and the boundary, we obtain (2).

Extending these ideas we have the following.

Lemma 3 Let $Q, Q^{\prime}$ be connected $d$-manifolds. Then

(A) if $Q, Q^{\prime}$ have nonempty boundary then

$$
\begin{aligned}
\left(Q \# Q^{\prime}\right) \times D^{1} & =\left(Q \times D^{1}\right) \amalg\left(Q^{\prime} \times D^{1}\right) \amalg\left(D^{2} \times S^{d-1}\right), \\
\mathcal{D}\left(Q \# Q^{\prime}\right) & =\mathcal{D} Q \# \mathcal{D} Q^{\prime} \#\left(S^{1} \times S^{d-1}\right), \\
\mathcal{T O} \mathcal{B}\left(Q \# Q^{\prime}\right) & =\mathcal{T} \mathcal{O B}(Q) \# \mathcal{T} \mathcal{O} \mathcal{B}\left(Q^{\prime}\right) \#\left(S^{2} \times S^{d-1}\right) ;
\end{aligned}
$$

(B) if $Q$ is closed but $Q^{\prime}$ has nonempty boundary then

$$
\begin{aligned}
\mathcal{D}\left(Q \# Q^{\prime}\right) & =Q \#(-Q) \# \mathcal{D} Q^{\prime}, \\
\mathcal{T} \mathcal{O B}\left(Q \# Q^{\prime}\right) & =\mathcal{G} Q \# \mathcal{T} \mathcal{O B}\left(Q^{\prime}\right) .
\end{aligned}
$$

${ }^{14}$ Unfortunately the Leibniz-Fico rule, $\mathcal{G}(M \times N)=(\mathcal{G} M \times N) \#(M \times \mathcal{G} N)$, generalizing (2) can only be valid when both $M$ and $N$ are spheres. 
Proof First observe that if $Q$ and $Q^{\prime}$ are disks, $Q \# Q^{\prime}$ is diffeomorphic to $D^{1} \times S^{d-1}$. Then, by the argument for property (e) preceding Lemma $1, Q \# Q^{\prime}$ can be obtained by identifying a disk in the boundary of $Q$ with a disk in the boundary of $D^{1} \times S^{d-1}$ and also a disk in the boundary of $Q^{\prime}$ with a disk in the other component of the boundary of $D^{1} \times S^{d-1}$. This is not the connected sum along the boundary of those three manifolds because not all their boundaries are connected. However, this problem again disappears after multiplying by $D^{1}: Q \times D^{1}, Q^{\prime} \times D^{1}$ and the intermediate manifold $D^{2} \times S^{d-1}$ all have connected boundaries and we have the connected sum along the boundary claimed in (A).

Taking its boundary and that of its product with $D^{1}$ gives the rest of (A).

As for (B) just observe by (e) above that $Q \# Q^{\prime}=Q_{-1} \amalg Q^{\prime}$ and take products and boundaries.

\subsection{Cutting vertices}

Let $P$ be a simple polytope of dimension $d$ with $m$ facets and $P_{\mathrm{v}}$ be a simple polytope of dimension $d$ obtained from $P$ by cutting off one vertex $v$.

Let $Z_{\mathrm{v}}=Z\left(P_{\mathrm{v}}\right)$ be the corresponding connected manifold. ${ }^{15}$

We will show that the operation $Z_{\mathrm{v}}$ preserves connected sums of sphere products. However, the result is not simply connected since $P_{\mathrm{v}}$ is never dual 2-neighborly because the new created facet does not intersect any of the facets not adjacent to $v$. So it cannot be combined with the theorems of Section 1 . We can partially avoid this problem by the next construction.

Let $Z_{\mathrm{v}}^{\prime}$ be the manifold obtained by duplicating the coefficients of the new variable $x_{0}$ introduced in the construction of $Z_{\mathrm{v}}$. Notice that this notation is consistent with that of Section 1.

Theorem 2.1 (1) $Z_{v}$ is diffeomorphic to $Z \# Z \#\left(2^{m-d}-1\right)\left(S^{1} \times S^{d-1}\right)$.

(2) $Z_{v}^{\prime}$ is diffeomorphic to $\mathcal{G} Z \#\left(2^{m-d}-1\right)\left(S^{2} \times S^{d-1}\right)$.

So, if $Z$ is a connected sum of sphere products, any manifold obtained from it by repeatedly applying the operations $Z_{v}$ and $Z_{v}^{\prime}$ is also a connected sum of sphere products.

${ }^{15}$ This manifold, the manifold $Z_{\mathrm{e}}$ and any other one obtained by cutting a face of $Z$ can be expressed as an intersection of quadrics by intersecting $P$ with a half space that contains all the vertices of $P$ that do not belong to the face, as explained in Section 0.2. 
Proof We look at $Z$ as the quotient of $P \times \mathbb{Z}_{2}^{m}$ by making identifications on the facets of $P$. Assume that the vertex $v$ lies in facets $F_{1}, \ldots, F_{d}$ of $P$ and the rest of the facets are $F_{d+1}, \ldots, F_{m}$. Call $F_{0}$ the new facet of $P_{\mathrm{v}}$ left by the removal of a small simplex $\Delta$ around $v$.

Then $Z_{\mathrm{v}}$ is obtained from $P_{\mathrm{v}} \times \mathbb{Z}_{2}^{m+1}$ by making the identifications on all of its facets. Let $Z_{\mathrm{v}+}$ be the manifold with boundary obtained by making all identifications on the facets of $P_{v} \times \mathbb{Z}_{2}^{m}$, except for $F_{0}$. Then, in the notation of Section 2.1, we have

$$
\begin{aligned}
& Z_{\mathrm{v}}=\mathcal{D} Z_{\mathrm{v}+}, \\
& Z_{\mathrm{v}}^{\prime}=\mathcal{T} \mathcal{O} \mathcal{B}\left(Z_{\mathrm{v}+}\right) .
\end{aligned}
$$

Now, $Z_{\mathrm{v}+}$ is $Z$ minus $U$ where $U$ is obtained from $\Delta \times \mathbb{Z}_{2}^{m}$ by making the identifications on the subsets $\Delta \cap F_{i}$, where $F_{i}$ are the facets of $P$.

If we make the identifications on $\Delta \times \mathbb{Z}_{2}^{d}$ on the facets $\Delta \cap F_{i}$ for $i=1, \ldots, d$ we obtain a disk ${ }^{16}$ of dimension $d, D^{d}$. On the product $D^{d} \times \mathbb{Z}_{2}^{m-d}$ we still have to make identifications on the subsets $\Delta \cap F_{i}$ for $i=d+1 \ldots, m$, but these intersections are empty. Therefore $U=D^{d} \times \mathbb{Z}_{2}^{m-d}$ and $Z_{\mathrm{v}+}$ is $Z$ with $2^{m-d}$ disks removed.

Now we use the following lemma.

Lemma Let $M^{d}$ be a connected manifold and $M_{-k}$ be obtained from $M$ by removing $k$ open disks. Then

(1) $M_{-k} \times D^{1}=\left(M_{-1} \times D^{1}\right) \amalg(k-1)\left(D^{2} \times S^{d-1}\right)$;

(2) $\mathcal{D}\left(M_{-k}\right)=M \#(-M) \#(k-1)\left(S^{1} \times S^{d-1}\right)$;

(3) $\mathcal{T O B}\left(M_{-k}\right)=\mathcal{G} M \#(k-1)\left(S^{2} \times S^{d-1}\right)$.

Proof Since $M$ is connected, we can assume all the open disks lie in the interior of a closed disk and therefore $M_{-k}$ can be seen as $M_{-1}$ with $k-1$ copies of $D^{1} \times S^{d-1}$ attached along disks in its boundary. This is not a connected sum along the boundary, since the boundary of $D^{1} \times S^{d-1}$ is not connected, but after multiplying times $D^{1}$ this problem disappears and $M_{-k} \times D^{1}$ is the connected sum claimed in (1). Taking its boundary we get part (2) and taking its boundary after multiplying again by $D^{1}$ gives part (3). So the lemma is proved and so are parts (1) and (2) of Theorem 2.1.

The last part is obvious for the operation $Z_{\mathrm{v}}$ and it follows from Fico's Lemmata (Lemma 2) for the operation $Z_{\mathrm{v}}^{\prime}$.

${ }^{16}$ If we had made identification on $\Delta \times \mathbb{Z}_{2}^{d+1}$ on all the facets we would have obtained the sphere $S^{d}$. 
Remarks Although the combinatorial type of $P_{\mathrm{v}}$ depends on which vertex is cut off, the diffeomorphism type of $Z_{v}$ does not. This gives many examples of different polytopes that produce the same manifold $Z$.

The analogous facts about $Z^{\mathbb{C}}$ appear in [8].

In particular, if $P$ is obtained from a simplex by successively cutting off vertices then $Z(P)$ is a connected sum of sphere products. Such a polytope is a dual stack polytope, and its dual can be obtained from a simplex by successively building up pyramids on facets.

Its diffeomorphism type depends only on the number of times each operation is applied and not on which vertices, or in which order, they are applied. As an exercise, write the formula for the effect on the manifold $Z$ of successively cutting off $n$ vertices from $P$.

Part (2) of Theorem 2.1 can be combined with the theorems of Section 1 to obtain that connected sums are preserved when applying repeatedly the $Z^{J}$ and the $Z_{\mathrm{v}}^{\prime}$ constructions. A more complete version of this will appear as Theorem 2.4 below.

Nevertheless, one would like to have a much more general vertex cutting result. We do not know, for example, if $Z^{\mathbb{C}}\left(P_{\mathrm{v}}\right)$ is a connected sum of sphere products whenever $Z^{\mathbb{C}}(P)$ is.

More generally, we can make the following conjecture.

Conjecture $Z^{\mathbb{C}}\left(P_{\mathrm{v}}\right)$ is diffeomorphic to $\mathcal{G}\left(Z^{\mathbb{C}}(P)\right) \underset{j=1}{\#-d}\left(\begin{array}{c}m-d \\ j\end{array}\right)\left(S^{j+2} \times S^{d+m-j-1}\right)$. We now prove it under a restriction on the number of facets of the polytope.

Theorem 2.2 If $P$ is a polytope of dimension $d$ with $m<3 d$ facets then the above conjecture is true.

Proof We will follow the same strategy as in the previous results, although now the steps are less elementary.

(1) The manifold $Z^{\mathbb{C}}(P)$ is obtained from $P \times\left(S^{1}\right)^{m}$ by making identifications on the $m$ facets. To obtain $Z^{\mathbb{C}}\left(P_{\mathrm{v}}\right)$ we have to multiply $P_{\mathrm{v}}$ by $\left(S^{1}\right)^{m+1}$ and make the identifications on the $m+1$ facets. Let $Z_{+}^{\mathbb{C}}\left(P_{\mathrm{v}}\right)$ be the manifold with boundary obtained from the product $\left(P_{\mathrm{v}}\right) \times\left(S^{1}\right)^{m}$ by making the identifications on all the facets 
except the new facet 0 . Then $Z^{\mathbb{C}}\left(P_{\mathrm{v}}\right)$ is obtained from $Z_{+}^{\mathbb{C}}\left(P_{\mathrm{v}}\right)$ by multiplying by $S^{1}$ and making the identifications on its boundary. In the notation of Section 2.1 this means

$$
Z^{\mathbb{C}}\left(P_{\mathrm{v}}\right)=\mathcal{T O} \mathcal{O}\left(Z_{+}^{\mathbb{C}}\left(P_{\mathrm{v}}\right)\right) .
$$

So we have to understand $Z_{+}^{\mathbb{C}}\left(P_{\mathrm{v}}\right) \times D^{1}$. Now $Z_{+}^{\mathbb{C}}\left(P_{\mathrm{v}}\right)$ is obtained from $Z^{\mathbb{C}}(P)$ by removing the manifold $U$ obtained from $\Delta^{d} \times\left(S^{1}\right)^{m}$ by making the same identifications as for $Z^{\mathbb{C}}(P)$, where $\Delta^{d}$ is the simplex removed to create the new facet 0 . If we make the identifications on $\Delta^{d} \times\left(S^{1}\right)^{d}$ corresponding to the facets of $\Delta^{d}$ that are part of facets of $P$ we obtain the disk $D^{2 d}$. On the product $\Delta^{d} \times\left(S^{1}\right)^{m}$ we still have to make identifications on the intersections with the $m-d$ facets not adjacent to the vertex $v$ but these intersections are empty. Therefore $U=D^{d} \times\left(S^{1}\right)^{m-d}$ and $Z_{+}^{\mathbb{C}}\left(P_{\mathrm{v}}\right)$ is $Z^{\mathbb{C}}(P)$ with a thickened $(m-d)$-torus removed.

(2) This $(m-d)$-torus can be contracted to a point in $Z^{\mathbb{C}}(P)$ because it is an orbit of the action of $T^{m}$ (see Section 0.2). Since $2(m-d)<m+d$ it is isotopic ${ }^{17}$ to an $(m-d)$-torus inside an open disk in $Z^{\mathbb{C}}(P)$. Therefore

$$
Z_{+}^{\mathbb{C}}\left(P_{\mathrm{v}}\right)=Z^{\mathbb{C}}(P) \#\left(S^{m+d} \backslash\left(T^{m-d} \times \operatorname{int}\left(D^{2 d}\right)\right)\right) .
$$

Again, since $2(m-d)<m+d$ we can apply Theorem A2.3 to the last summand and Lemma 2 of Section 2.1 to the whole sum to obtain the Theorem.

Without the restriction on the number of facets of $P$, it can be proved that the $(m-d)-$ torus can be engulfed by an open disk in $Z^{\mathbb{C}}(P)$. To prove the general conjecture, one would need to prove that this torus is in some sense standard inside that disk and some version of Theorem A2.3 for that type of standard torus.

A more general formula can be conjectured when the construction $Z^{J}$ is immediately applied after cutting off a vertex. Again a partial case can be deduced from Theorem A2.2.

We need also, of course, a way to include all the nonsimply connected manifolds into the picture.

Example (The truncated cube) We will study now the cube $C=I \times I \times I$ and the truncated cube $C_{\mathrm{v}}$.

Now we have

$$
\begin{aligned}
Z(C) & =S^{1} \times S^{1} \times S^{1}, \\
Z^{\mathbb{C}}(C) & =S^{3} \times S^{3} \times S^{3} .
\end{aligned}
$$

${ }^{17}$ This follows from Haefliger's isotopy theorem [19, Theorem 1(b)]. 


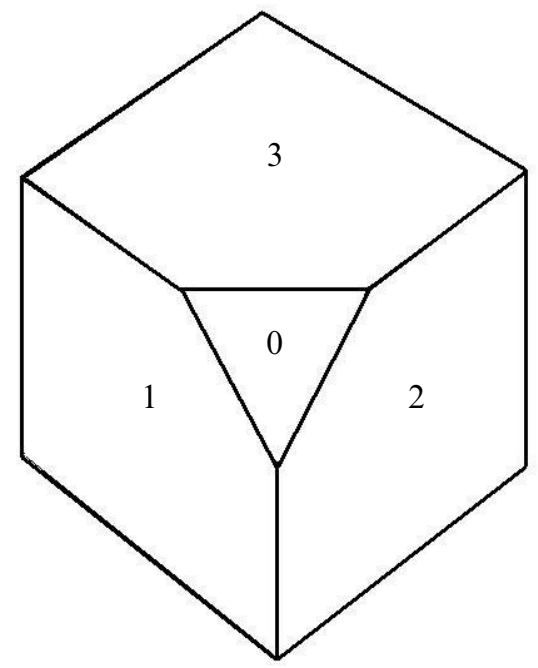

From Theorem 2.1 we know that

$$
Z\left(C_{\mathrm{v}}\right)=\left(S^{1} \times S^{1} \times S^{1}\right) \#\left(S^{1} \times S^{1} \times S^{1}\right) \# 7\left(S^{1} \times S^{2}\right) .
$$

From Theorem 2.2 we know that

$$
Z^{\mathbb{C}}\left(C_{\mathrm{v}}\right)=\mathcal{G}\left(S^{3} \times S^{3} \times S^{3}\right) \# 3\left(S^{3} \times S^{7}\right) \# 3\left(S^{4} \times S^{6}\right) \#\left(S^{5} \times S^{5}\right) .
$$

It follows from the cohomology ring of $\mathcal{G}\left(S^{3} \times S^{3} \times S^{3}\right)$ that this manifold does not decompose into a nontrivial connected sum: by the argument of the Proposition in Section 3, the 3-dimensional generators would have to lie in one of the summands and so would the rest of the generators of dimension less that 10 since they are related by nontrivial products with the first ones, so one of the summands must be a homotopy sphere. This shows that we have reached the whole decomposition of $Z^{\mathbb{C}}\left(C_{\mathrm{v}}\right)$ as a connected sum.

This answers another question in [8, page 111]. It will prove useful in Section 3.

We can also describe the topology of the manifolds associated with the truncated $d$-cube. In fact, Theorem 2.2 gives us the topology of $Z^{\mathbb{C}}$ of an iterated truncation of up to $d$ vertices of the $d$-cube.

\subsection{Cutting edges and other faces}

Let $P$ be a simple polytope of dimension $d$ and $m$ facets and $P_{\mathrm{e}}$ be a simple polytope of dimension $d$ obtained from $P$ by cutting off one edge $e$. 
Let $Z_{\mathrm{e}}=Z\left(P_{\mathrm{e}}\right)$. Again, the resulting manifold is not simply connected, but once more we can prove that it becomes simply connected after applying to it the construction $Z^{\prime}$ based on the new facet of $P_{\mathrm{e}}$. Let $Z_{\mathrm{e}}^{\prime}$ be that manifold.

Theorem 2.3 If $Z$ is 1-connected (or, equivalently, if $P$ is dual 2-neighborly) then

(1) $Z_{e}$ is diffeomorphic to

$$
Z \# Z \#\left(2^{m-d-1}\right)\left(S^{2} \times S^{d-2}\right) \#\left(2^{m-d-1}-1\right)\left(S^{1} \times S^{d-1}\right),
$$

(2) $Z_{e}^{\prime}$ is diffeomorphic to

$$
\mathcal{G}(Z) \#\left(2^{m-d-1}\right)\left(S^{3} \times S^{d-2}\right) \#\left(2^{m-d-1}-1\right)\left(S^{2} \times S^{d-1}\right) .
$$

Proof We look at $Z$ as the quotient of $P \times \mathbb{Z}_{2}^{m}$ by making identifications on the facets of $P$. Denote by $F_{1}, \ldots, F_{m}$ these facets, where $F_{1}, \ldots F_{d+1}$ are the ones touched by $e$. Call $F_{0}$ the new facet of $P_{\mathrm{e}}$ left by the removal of a regular neighborhood $e \times \Delta^{d-1}$ of $e$.

Then $Z_{\mathrm{e}}$ is obtained from $P_{\mathrm{e}} \times \mathbb{Z}_{2}^{m+1}$ by making the identifications on its $m+1$ facets. Let $Z_{\mathrm{e}+}$ be obtained from $P_{\mathrm{e}} \times \mathbb{Z}_{2}^{m}$, by making identifications on the facets $F_{1}, \ldots, F_{m}$. Then clearly we have

$$
Z_{\mathrm{e}}=\mathcal{D}\left(Z_{\mathrm{e}+}\right)
$$

Also, $Z_{\mathrm{e}}^{\prime}$ is obtained from $P_{\mathrm{e}} \times \mathbb{Z}_{2}^{m} \times S^{1}$ by making the identifications on its facets, so, in the notation of Section 2.1,

$$
Z_{\mathrm{e}}^{\prime}=\mathcal{T} \mathcal{O B}\left(Z_{\mathrm{e}+}\right)
$$

So we have to describe $Z_{\mathrm{e}+} \times D^{1}$ and $Z_{\mathrm{e}+} \times D^{2}$ in order to know their boundaries.

Now $Z_{\mathrm{e}+}$ is $Z$ minus $U$ where $U$ is obtained from $e \times \Delta^{d-1} \times \mathbb{Z}_{2}^{m}$ by making the identifications on its intersections with the facets $F_{1}, \ldots, F_{m}$ of $P$.

If we make first the identifications on $e \times \Delta^{d-1} \times \mathbb{Z}_{2}^{d+1}$ on all its facets we obtain the product $S^{1} \times S^{d-1}$, but if we only do it on those facets that are part of the facets of $P$ (ie, excluding the facet 0 ) we obtain $S^{1} \times D^{d-1}$. On the product $e \times \Delta^{d-1} \times \mathbb{Z}_{2}^{m}$ we still have to make identifications on the intersections with the facets $i$ for $i=d+2, \ldots, m$ but these intersections are empty. Therefore $U=S^{1} \times D^{d-1} \times \mathbb{Z}_{2}^{m-d-1}$ and $Z_{\mathrm{e}+}$ is $Z$ with $2^{m-d-1}$ copies of $S^{1} \times D^{d-1}$ removed.

Then we have the following lemma. ${ }^{18}$

${ }^{18}$ Recall the notation of Section 2.1: $M_{-1}$ denotes $M$ minus an open disk. For dimension 3 the lemma is not true, but the theorem is, since the only dual 2-neighborly polytope is the simplex. 
Lemma Let $M^{d}$ be a simply connected manifold of dimension at least 4 and $M_{\sim k}$ be obtained from $M$ by removing $k>0$ copies of $S^{1} \times D^{d-1}$.

Then $M_{\sim k} \times D^{1}$ is diffeomorphic to

$$
M_{-1} \times D^{1} \amalg k\left(D^{3} \times S^{d-2}\right) \amalg(k-1)\left(D^{2} \times S^{d-1}\right) .
$$

Proof Since $M$ is simply connected, we can assume all copies of $S^{1} \times D^{d-1}$ lie inside an open disk, so we have only to prove it for $M=S^{d}$.

In this case the result is true for $k=1$ (even before multiplying by $D^{1}$ ) since $S^{d}$ minus a copy of $S^{1} \times D^{d-1}$ is diffeomorphic to $D^{2} \times S^{d-2}$.

Inductively, $M_{\sim(k+1)}$ is obtained from $M_{\sim k}$ by removing one copy of $S^{1} \times D^{d-1}$ from it so $M_{\sim(k+1)}=M_{\sim k} \# M_{\sim 1}$.

Now Lemma 3(A) of Section 2.1 gives the induction step

$$
\begin{aligned}
M_{\sim(k+1)} \times D^{1} & =\left(M_{\sim k} \# M_{\sim 1}\right) \times D^{1} \\
& =\left(M_{\sim k} \times D^{1}\right) \amalg\left(M_{\sim 1} \times D^{1}\right) \amalg\left(D^{2} \times S^{d-1}\right) .
\end{aligned}
$$

If we substitute $M_{1} \times D^{1}$ by $D^{3} \times S^{n-2}$ and $M_{k} \times D^{1}$ by the induction hypothesis the Lemma is proved.

Applying it to $Z$ we obtain that $Z_{\mathrm{e}+} \times D^{1}$ is diffeomorphic to

$$
\left(Z_{-1} \times D^{1}\right) \amalg 2^{m-d-1}\left(D^{3} \times S^{d-2}\right) \#\left(2^{m-d-1}-1\right)\left(D^{2} \times S^{d-1}\right) .
$$

Taking its boundary we obtain (1). Taking the boundary of its product with $D^{1}$ we obtain (2).

Remarks (1) Although the combinatorial type of $P_{\mathrm{e}}$ depends on which edge is cut off, the diffeomorphism type of $Z_{\mathrm{e}}$ does not. This gives many new examples of different polytopes that produce the same manifold $Z$.

(2) If $Z$ is not simply connected then Theorem 2.3 and the previous observation are not true. For example, if $P$ is the triangular prism $\Delta^{2} \times I$, then by cutting off an edge we can obtain either the pentagonal book or the cube, whose corresponding manifolds are \#5( $\left.S^{1} \times S^{2}\right)$ and $S^{1} \times S^{1} \times S^{1}$, respectively. The reason lies in the fact that when the edge connects two facets of $P$ that do not intersect, then this edge produces an embedded $S^{1}$ that is not trivial in $H_{1}(Z)$ and therefore cannot be contained inside a disk. It remains to be clarified what happens in this case.

(3) One problem with part (1) is that it cannot be immediately iterated since the resulting manifold $Z_{\mathrm{e}}$ is not simply connected. 
Combining Theorem 2.3 with our previous theorems we get the following.

Theorem 2.4 If $Z=Z(P)$ is a connected sum of sphere products and is simply connected of dimension at least 5 , then any manifold obtained from $Z$ by repeatedly applying the $Z^{J}, Z_{v}^{\prime}$ and $Z_{e}^{\prime}$ constructions (any number of times each and in any order) is also a connected sum of sphere products. ${ }^{19}$

The basic manifold can be any sphere, any $Z(P)$ with free homology for a dualneighborly polytope (Theorems 1.3 and 1.4) or any simply connected manifold with $m-d=3$ of dimension at least 5 that is not a triple sphere product [26].

It is known that there are many dual neighborly polytopes in each dimension. ${ }^{20}$ If to these we add the infinite family stemming from each of them by applying the three constructions above (which produce polytopes which are not dual neighborly) we can conclude that there is a large number of polytopes $P$ for which $Z(P)$ is a connected sum of sphere products.

The theorems in this section can be generalized to the process of removing any simplicial face of $P$ but need, of course, stronger connectivity hypotheses.

One can conceive also results about removing nonsimplicial faces of $P$. It has been conjectured that any simple polytope can be realized as a face of a dual neighborly polytope, so in principle we need to consider the effect of cutting off any possible simple polytope.

\section{On the cohomology ring of $Z(P)$}

In [8] the cohomology ring of $Z^{\mathbb{C}}(P)$ is described (by an explicit rule) in terms of intersection products on the homology groups of unions of facets of $P$. A slightly different rule, valid for general moment-angle complexes, appears in [5]. We shall see now by examples that these rules must be drastically modified in the case of the manifolds $Z(P)$.

We will show that the cohomology rings of the manifolds associated to the truncated cube are not isomorphic as ungraded rings.

${ }^{19}$ This includes, of course, the corresponding moment-angle manifolds.

${ }^{20}$ In Grünbaum [18, page 129a], one can find very large lower bounds for the number of combinatorially different neighborly polytopes of a given dimension and number of vertices, "remarkably close" to the total number of the simple polytopes with the same data. A vague feeling among experts that most polytopes are neighborly is expressed in page 129 and a probabilistic approach is discussed in page $129 \mathrm{~b}$. 
We will consider the cohomology rings with $\mathbb{Z}_{2}$ coefficients. Since all the homology groups of the spaces involved are free, this will be enough to establish the difference without having to compute signs, so in fact we have a stronger statement. We have shown at the end of Section 2.2 that

$$
\begin{aligned}
Z\left(C_{\vee}\right) & =\left(S^{1} \times S^{1} \times S^{1}\right) \#\left(S^{1} \times S^{1} \times S^{1}\right) \# 7\left(S^{1} \times S^{2}\right), \\
Z^{\mathbb{C}}\left(C_{\vee}\right) & =\mathcal{G}\left(S^{3} \times S^{3} \times S^{3}\right) \# 3\left(S^{3} \times S^{7}\right) \# 3\left(S^{4} \times S^{5}\right) \#\left(S^{5} \times S^{5}\right) .
\end{aligned}
$$

We begin by describing the cohomology ring of the first summands of each manifold:

$$
X:=\left(S^{1} \times S^{1} \times S^{1}\right) \#\left(S^{1} \times S^{1} \times S^{1}\right), \quad Y:=\mathcal{G}\left(S^{3} \times S^{3} \times S^{3}\right) .
$$

First, $H^{*}(X)$ has

- one generator 1 in dimension 0 ;

- six generators $a_{1}, a_{2}, a_{3}, a_{1}^{\prime}, a_{2}^{\prime}, a_{3}^{\prime}$ in dimension 1 ;

- six generators $b_{1,2}, b_{1,3}, b_{2,3}, b_{1,2}^{\prime}, b_{1,3}^{\prime}, b_{2,3}^{\prime}$ in dimension 2 ;

- one generator $f$ (the fundamental class) in dimension 3 .

The multiplication rules are

$$
\begin{aligned}
a_{i} a_{j} & =b_{i, j} \quad \text { for } i \neq j, \\
a_{1} a_{2} a_{3} & =f, \\
a_{i}^{\prime} a_{j}^{\prime} & =b_{i, j}^{\prime} \quad \text { for } i \neq j, \\
a_{1}^{\prime} a_{2}^{\prime} a_{3}^{\prime} & =f,
\end{aligned}
$$

with all other products not derived from these being 0 .

On the other hand, $H^{*}(Y)$ has

- one generator 1 in dimension 0 ;

- three generators $A_{1}, A_{2}, A_{3}$ in dimension 3;

- three generators $A_{1}^{\prime}, A_{2}^{\prime}, A_{3}^{\prime}$ in dimension 4;

- three generators $B_{1,2}, B_{1,3}, B_{2,3}$ in dimension 6;

- three generators $B_{1,2}^{\prime}, B_{1,3}^{\prime}, B_{2,3}^{\prime}$ in dimension 7;

- one generator $F^{\prime}$ (the fundamental class) in dimension 10 .

(In this case a generator is denoted by $C^{\prime}$ when it is inherited from the element $C \times u$ in the cohomology of $\left(S^{3} \times S^{3} \times S^{3}\right) \times S^{1}$, where $u$ is the generator of $\left.H^{1}\left(S^{1}\right)\right)$. 
The multiplication rules are

$$
\begin{aligned}
A_{i} A_{j} & =B_{i, j} & & \text { for } i \neq j \\
A_{i} A_{j}^{\prime} & =B_{i, j}^{\prime} & & \text { for } i \neq j \\
A_{i} A_{j} A_{k}^{\prime} & =F^{\prime} & & \text { for } i, j, k \text { all different }
\end{aligned}
$$

with all other products not derived from these being 0 .

Observe in particular that

$$
A_{i}^{\prime} A_{j}^{\prime}=0
$$

for all $i, j$, simply because the product lies in $H^{8}(Y)=0$.

This is the main difference with the previous ring: three indecomposable, linearly independent elements have all their products equal to zero. To make this idea into a formal proof of nonisomorphism we have to work modulo the decomposable elements.

In both rings there is the maximal ideal $\mathcal{M}$ of nonunits, spanned by all the generators except 1 . The square of this ideal $\mathcal{M}^{2}$ is generated by the decomposable elements and its cube is generated by the fundamental class. There is an induced multiplication

$$
M: \mathcal{M} / \mathcal{M}^{2} \times \mathcal{M} / \mathcal{M}^{2} \rightarrow \mathcal{M}^{2} / \mathcal{M}^{3} .
$$

An ungraded ring isomorphism between the two rings would have to preserve the above ideals and multiplication.

Proposition For the ring $H^{*}(X)$, any three elements in $\mathcal{M} / \mathcal{M}^{2}$ all whose products are zero in $\mathcal{M}^{2} / \mathcal{M}^{3}$ must be linearly dependent.

Proof Let

$$
x_{j}=\sum_{i=1}^{3} \lambda_{j}^{i} a_{i}+\sum_{i=1}^{3} \mu_{j}^{i} a_{i}^{\prime}
$$

for $i=1, \ldots, 3$ be (representatives of) such elements. Then

$$
x_{j} x_{k}=\sum_{r<s}\left(\lambda_{j}^{r} \lambda_{k}^{s}+\lambda_{j}^{s} \lambda_{k}^{r}\right) b_{r, s}+\sum_{r<s}\left(\mu_{j}^{r} \mu_{k}^{s}+\mu_{j}^{s} \mu_{k}^{r}\right) b_{r, s}^{\prime} .
$$

The fact that this product is 0 means that all $2 \times 2$ determinants of the matrices $\lambda_{j}^{i}$ and $\mu_{j}^{i}$ are 0 . This means that in each of these matrices there is at most one linearly independent column and that the matrix of coefficients of the $x_{i}$, which is formed by the columns of those two, has rank at most 2. Thus the Proposition is proved. 
To get the complete rings of $Z\left(C_{\mathrm{v}}\right)$ and $Z^{\mathbb{C}}\left(C_{\mathrm{v}}\right)$ one has to add in each case to the corresponding previous one 7 couples of elements $u_{i}, v_{i}$ such that each product $u_{i} v_{i}$ is the fundamental class and the product of each $u_{i}, v_{i}$ with all the rest of generators is 0 . We have the following.

Theorem 3.1 The rings $H^{*}\left(Z\left(C_{v}\right), \mathbb{Z}_{2}\right)$ and $H^{*}\left(Z^{\mathbb{C}}\left(C_{v}\right), \mathbb{Z}_{2}\right)$ are not isomorphic as ungraded rings.

Proof We follow the same lines as for the previous proposition: Since the fundamental class is in $\mathcal{M}^{3}$ this means that $H^{*}\left(Z^{\mathbb{C}}\left(C_{\mathrm{v}}\right)\right)$ has 17 generators in $\mathcal{M} / \mathcal{M}^{2}$ all whose products are 0 under the induced multiplication $M$. If in the case of $H^{*}\left(Z\left(C_{\mathrm{v}}\right), \mathbb{Z}_{2}\right)$ we have 17 elements in $\mathcal{M} / \mathcal{M}^{2}$ for which all products under $M$ are 0 , we can express them in terms of the 20 generators $a_{i}, a_{i}^{\prime}, u_{i}, v_{i}$ by a $17 \times 20$ matrix. By the same computation as above, at most two of the first six columns of this matrix are independent, so the matrix has at most rank 16 and the 17 elements are not linearly independent. This proves the Theorem.

Remarks (1) The analogues of the product rules in [6;8] for the ring $H^{*}\left(Z^{\mathbb{C}}(P)\right)$ are not valid in general for the ring $H^{*}(Z(P))$.

(2) Theorem 3.1 contradicts the results on the cohomology ring of the complement of a real coordinate subspace arrangements in [23, Remark 4.1] since everyone of our manifolds has the homotopy type of such a complement. Actually, the example where $P$ is a pentagon (the truncated square) already shows that the product rule has to be modified in the real case. But for the pentagon the cohomology rings over $\mathbb{Z}_{2}$ turn out to be isomorphic as ungraded rings, even if the natural additive isomorphism does not preserve the ring structure. But in our example there is no possible ring isomorphism at all, and the same happens for any truncated cube of dimension greater than 2. We thank Taras Panov for pointing out that another example can be found in Gasharov, Peeva and Welker [16]. This example is a subspace arrangement that does not correspond to a convex polytope.

(3) It can be shown that the cohomology rings of $Z^{\mathbb{C}}(P)$ and $Z^{\mathbb{C}}\left(P^{\prime}\right)$ are isomorphic as ungraded rings (see Bahri, Bendersky, Cohen and the first author [3]); this should also be a consequence of the other known descriptions of the cohomology rings $[8 ; 10]$. For example, if $P$ is a product of two simplices then $Z^{\mathbb{C}}(P)=S^{2 p-1} \times S^{2 q-1}$ and $Z^{\mathbb{C}}\left(P^{\prime}\right)=S^{2 p+1} \times S^{2 q-1}$ and the ungraded cohomology rings are isomorphic, with generators $\{1, x, y, x y\}$. Or, if $Z^{\mathbb{C}}(P)$ is a connected sum of sphere products then $Z^{\mathbb{C}}\left(P^{\prime}\right)$ is also one, with the same number of summands, but where inside each summand one of the factors has risen its dimension by two. 
(4) Theorem 3.1 implies that this also cannot be true in general for the cohomology rings of $Z$ and $Z^{\prime}$, not even when taken with $\mathbb{Z}_{2}$ coefficients, since the passage from $Z(P)$ to $Z^{\mathbb{C}}(P)$ goes through the iteration of the operation $Z^{\prime}$.

We claim that the correct rules are as follows: Let $L$ be a collection of facets of $P$, we denote by $P_{L}$ the union of the facets of $P$ in $L$.

Intersection product There is an isomorphism

$$
H_{i}(Z) \approx \bigoplus_{L} H_{i}\left(P, P_{L}\right)
$$

so that the intersection of two homology classes in $Z$ is given by the sum of the intersection products on the right hand side

$$
H_{i}\left(P, P_{L}\right) \otimes H_{j}\left(P, P_{J}\right) \rightarrow H_{i+j-d}\left(P, P_{L \cap J}\right) .
$$

Cup product There is an isomorphism

$$
H^{i}(Z) \approx \bigoplus_{L} H^{i}\left(P, P_{L}\right)
$$

so that the cup product of two cohomology classes in $Z$ is given by the sum of the cup products on the right hand side

$$
H^{i}\left(P, P_{L}\right) \otimes H^{j}\left(P, P_{J}\right) \rightarrow H^{i+j}\left(P, P_{L \cup J}\right) .
$$

In other words, the rule that a product must be 0 unless $L$ and $J$ satisfy a strict set-theoretical condition, must be dropped.

Details and extensions will appear in a forthcoming article.

\section{Appendix A1: Recognizing a connected sum of sphere prod- ucts}

Recall that, after Thom, Smale and Milnor, the best way to identify a compact manifold $M$ is to study an adequate compact manifold $Q$ whose boundary is $M$. For example, if $M^{n}$ is a homotopy sphere, we cannot tell if it is diffeomorphic to the standard sphere $S^{n}$ by looking at the usual topological invariants of $M$ : since these are homotopy invariants there is nothing to look at. But we know that $M$ is the boundary of some manifold $Q$. The first thing to ask is if $Q$ is parallelizable. If there is no such parallelizable $Q$ then $M$ is not standard since $S^{n}$ bounds a disk. If one finds a $Q$ that is parallelizable, then one can decide if $M$ is standard by studying topological invariants of $Q$ [21]. 
Now a connected sum of sphere products

$$
\underset{i=1}{k}\left(S^{p_{i}} \times S^{d-p_{i}}\right)
$$

is the boundary of the connected sum along the boundary

$$
\coprod_{i=1}^{k}\left(S^{p_{i}} \times D^{d-p_{i}+1}\right),
$$

which has the following properties: It is simply connected if $1<p_{i}$, has simply connected boundary if $1<p_{i}<d-2$, its homology groups are free and bases of them can be represented by disjoint embedded spheres with trivial normal bundle.

To determine such a connected sum it is enough to verify the following conditions.

Theorem A1.1 Let $Q$ be a manifold with boundary satisfying

(1) $Q$ is of dimension $d+1 \geq 6$;

(2) $Q$ is simply connected with simply connected boundary;

(3) $H_{i}(Q)$ is free with basis $\alpha_{i, j}$ for all $i$ and $H_{i}(Q)=0$ for $i \geq d-1$;

(4) there is a collection $\left\{S_{j}^{i}\right\}$ of disjoint embedded spheres with trivial normal bundle inside $Q$ that represent the basis elements $\alpha_{i, j}$ of $H_{i}(Q)$.

Then $Q$ is diffeomorphic to a connected sum along the boundary

$$
\coprod_{i=1}^{k}\left(S^{p_{i}} \times D^{d-p_{i}+1}\right)
$$

and therefore $\partial Q$ is diffeomorphic to

$$
\underset{i=1}{k}\left(S^{p_{i}} \times S^{d-p_{i}}\right) .
$$

Proof For every sphere $S_{j}^{i}$ take a closed product neighborhood $U_{i, j}=S_{j}^{i} \times D^{d-i}$ in such a way that they are all disjoint. Connect all $U_{i, j}$ by a minimal collection of thin tubes and call $Q_{1}$ the union of all the $U_{i, j}$ and their connecting tubes, which we can assume is contained in the interior of $Q$. Then $Q_{1}$ is a connected sum of the required form for $Q$ and we will prove that these two manifolds are diffeomorphic. Let $H$ be equal to $Q$ minus the interior of $Q_{1}$, so that $H$ is a cobordism between the manifolds $\partial Q$ and $\partial Q_{1}$.

Now $Q_{1}, \partial Q_{1}$ are simply connected because all the spheres $S_{j}^{i}$ have dimension $2 \geq i \geq d-2$ and so is $H$ because $Q$ is simply connected and every loop in $H$ bounds 
a disk in $Q$ that can be made disjoint from $Q_{1}$ since the spheres have codimension greater than 2. Now $H_{i}\left(H, \partial Q_{1}\right)=H_{i}\left(Q, Q_{1}\right)=0$ for all $i$ because the inclusion of $Q_{1}$ in $Q$ induces isomorphisms of homology groups. Therefore $H$ is a simply connected $h$-cobordism of dimension at least 6 between simply connected manifolds and so is a product by the $h$-cobordism theorem. This proves that $Q$ is diffeomorphic to $Q_{1}$ and the theorem is proved.

\section{Appendix A2: Complements of sphere products in spheres}

We will work in the unit sphere $S^{n}$ in $\mathbb{R}^{n+1}$ and assume we have a decomposition

$$
\mathbb{R}^{n+1}=\mathbb{R}^{n_{1}+1} \times \cdots \times \mathbb{R}^{n_{k}+1} \times \mathbb{R}^{q} .
$$

We have then $n=\sum_{i=1}^{k} n_{i}+q+k-1$ and we will denote an element in $\mathbb{R}^{n+1}$ as a $(k+1)$-tuple $\left(X_{1}, \ldots, X_{k}, Y\right)$ with $X_{i} \in \mathbb{R}^{n_{i}+1}$ and $Y \in \mathbb{R}^{q}$.

We will consider the product of spheres

$$
\mathcal{P}=S^{n_{1}} \times \cdots \times S^{n_{k}} \times\{0\}
$$

given by the equations

$$
X_{i} \cdot X_{i}=\frac{1}{k}, \quad Y=0 .
$$

So $\mathcal{P}$ has dimension $\sum_{i=1}^{k} n_{i}$ and codimension $q+k-1$.

Within each sphere $S^{n_{i}}$ we consider the base point $p_{i}=(1 / \sqrt{k}, 0, \ldots, 0)$. We denote by $p$ the point $\left(p_{1}, \ldots, p_{k}, 0\right) \in \mathbb{R}^{n+1}$.

We are interested in the complement of an open tubular neighborhood of $\mathcal{P}$ in $S^{n}$. We will denote this complement by $\mathcal{E}$.

Let $I=\{1, \ldots, k\}$. If $J \subset I$ we denote by $|J|$ the number of elements of $J$ and by $n_{J}$ the sum $\sum_{i \in J} n_{i}$.

We will make use of the subproduct $\mathcal{P}_{J}$ of $\mathcal{P}$ defined by the equations

$$
\begin{aligned}
X_{i} & =p_{i}, \quad i \notin J, \\
Y & =0 .
\end{aligned}
$$

So $\mathcal{P}_{J}$ is diffeomorphic to the product $\prod_{i \in J} S^{n_{i}}$ of dimension $n_{J}$, the rest of the coordinates having fixed values.

If $n_{i}>0$ for $i=1, \ldots, k$ then $\mathcal{P}$ is connected and its reduced homology groups are free with a generator of dimension $n_{J}>0$ represented by the fundamental class of $\mathcal{P}_{\boldsymbol{J}}$ for every nonempty $J \subset I$. 
We will show that if $n_{i}>0$ for $i=1, \ldots, k$ we have Alexander dual generators for the homology of $\mathcal{E}$ represented by embedded round spheres. The Alexander dual of a single sphere $S^{n_{i}}$ will be a great sphere of dimension $n-n_{i}-1$ lying in a complementary subspace. The Alexander dual of the products of spheres will be spheres that become smaller and more slanted as the number of factors grows. The Alexander dual of the whole product $\mathcal{P}$ will be a small sphere which is a fiber of its spherical normal bundle.

A schematic representation of the construction is the following.

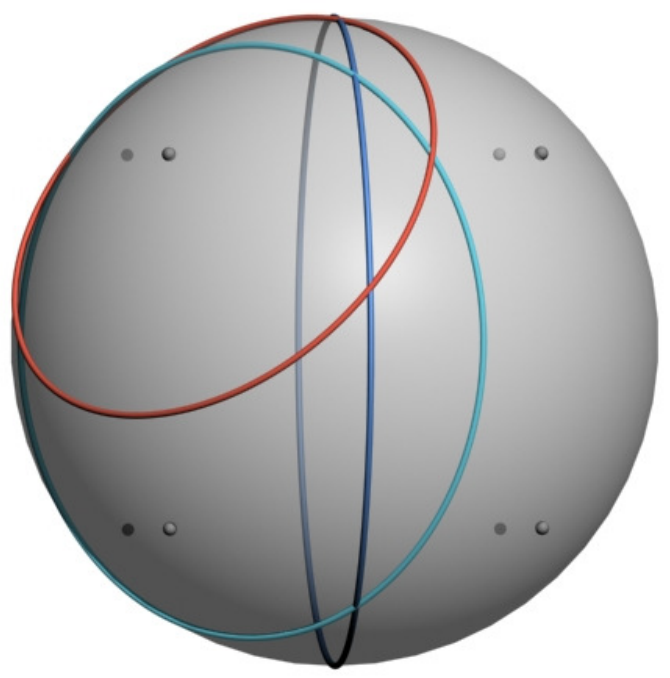

Here we have the picture for $n=2, k=3, n_{1}=n_{2}=0$ (a case not covered by the hypothesis of Theorem A2.1 below). The product $\mathcal{P}=S^{0} \times S^{0} \times S^{0}$ is given by the 8 marked points. The vertical great circle is the Alexander dual of an $S^{0}$ given by one point on each vertical hemisphere. The smaller vertical circle is the Alexander dual of an $S^{0} \times S^{0}$ consisting of the points in the upper hemisphere since the small disk this circle bounds contains exactly one of its points. The smallest slanted circle is the Alexander dual of the whole product, since it surrounds just one of its points.

Theorem A2.1 Assume $n_{i}>0$ for $i=1, \ldots, k$. Then there is a basis for the homology of $\mathcal{E}$ represented by embedded round spheres. This basis is in each dimension the Alexander dual basis of the basis $\mathcal{P}_{J}$ of the reduced homology of $\mathcal{P}$ in the corresponding dimension. 
Proof The result is well-known for $k=1$ so we assume $k>1$.

We will consider for each $J \subset I$ the following subspaces of $\mathbb{R}^{n+1}$.

Let $L_{J}$ be the vector subspace of $\mathbb{R}^{n+1}$ of codimension $n_{J}$ consisting of those points $(X, Y)$ such that, for $i \in J, X_{i}$ is a multiple of $p_{i}$ :

$$
X_{i}=t_{i} p_{i}, \quad \text { for } i \in J .
$$

Inside its unit sphere $L_{J} \cap S^{n}$ we consider the set $D_{J}$ of points satisfying

$$
\sum_{i \in J} t_{i} \geq|J|-1
$$

and the set $S_{J}$ of points satisfying

$$
\sum_{i \in J} t_{i}=|J|-1
$$

Since the function $\sum_{i \in J} t_{i}$ takes at $p \in L_{J} \cap S^{n}$ the value $|J|$, it follows that

(1) $D_{J}$ is a disk with nonempty interior in $L_{J} \cap S^{n}$;

(2) $S_{J}$ is a round sphere which is the boundary of $D_{J}$.

We claim that the $S_{J}$ represent the Alexander duals of the basis $\mathcal{P}_{J}$ of the homology of $\mathcal{P}$. We show this by proving that for each $J \neq \varnothing$,

(A) $S_{J}$ is contained in $\mathcal{E}$;

(B) the disk $D_{J}$ in $S^{n}$ whose boundary is $S_{J}$ intersects $\mathcal{P}_{J}$ transversely in exactly the point $p$;

(C) for any $J^{\prime} \neq J$ with $n_{J}=n_{J^{\prime}}$ the intersection number of $D_{J}$ with $\mathcal{P}_{J^{\prime}}$ is zero.

To prove (A) and (B) first observe that a point $(X, Y) \in \mathcal{P} \cap\left(L_{J} \cap S^{n}\right)$ must satisfy $Y=0$ and both $X_{i} \cdot X_{i}=\frac{1}{k}$ and $X_{i}=t_{i} p_{i}$ for $i \in J$, so $t_{i}= \pm 1$. If $\sum_{i \in J} t_{i} \geq|J|-1$ then $t_{i}=1$ and $X_{i}=p_{i}$ for $i \in J$ and so the preceding sum is actually $|J|$. This proves (A): $S_{J} \cap \mathcal{P}=\varnothing$. If, further, $(X, Y) \in \mathcal{P}_{\mathcal{J}}$ then $X_{i}=p_{i}$ also for $i \notin J$ so $X=p$ and we have proved a part of (B): $D_{J} \cap \mathcal{P}_{J}=\{p\}$.

To verify that the intersection is transversal just observe that the normal space to $\mathcal{P}_{\boldsymbol{J}}$ at $p$ is exactly $L_{J}$. Therefore, $\mathcal{P}_{J}$ and $L_{J}$ intersect transversely at $p$ in $\mathbb{R}^{n+1}$. It follows that $\mathcal{P}_{J}$ and $L_{J} \cap S^{n}$ also intersect transversely in $S^{n}$ at $p \in D_{J}$ and (B) is proved.

To prove (C) in each dimension first observe that if $J \subset J^{\prime}$ they would be equal since $n_{J}=n_{J^{\prime}}$ and by hypothesis every $n_{i}$ is positive. Then there is an $i \in J$ not in $J^{\prime}$ so 
$X_{i}=p_{i}$ both in $D_{J}$ and in $\mathcal{P}_{J^{\prime}}$. Now if we take $q_{i} \in \mathbb{R}^{n_{i}+1}$ close to $p_{i}$ with $q_{i} \neq p_{i}$ and $q_{i} \cdot q_{i}=k$ (which is possible because $n_{i}$ is positive) we get a new product $\mathcal{P}_{J^{\prime}}^{\prime}$ by changing the equation $X_{i}=p_{i}$ into $X_{i}=q_{i}$. So we have $D_{J} \cap \mathcal{P}_{J^{\prime}}^{\prime}=\varnothing$. Since $\mathcal{P}_{J^{\prime}}^{\prime}$ is close and isotopic to $\mathcal{P}_{J^{\prime}}$ we have proved (C).

So the linking number of $S_{J}$ with $\mathcal{P}_{J^{\prime}}$ is zero and Theorem A2.1 is proved.

For $k>1$ we cannot apply to $\mathcal{E}$ Theorem A1.1 characterizing a connected sum along the boundary (and in fact it is not, since its boundary is a product of at least three spheres). But we can apply it to $\mathcal{E} \times D^{1}$ since the spheres $S_{J}$ can be embedded with different values of the coordinate in $D^{1}$ so they are disjoint, represent a basis of its homology and have codimension at least 3 and dimension at least 2 if $q+k \geq 4$. As a consequence we have the following.

Theorem A2.2 If $q+k \geq 4$, then

(A) $\mathcal{E} \times D^{1}$ is diffeomorphic to

$$
\coprod_{\varnothing \neq J \subset I}\left(S^{n-n_{J}-1} \times D^{n_{J}+2}\right)
$$

(B) the double of $\mathcal{E}, \mathcal{D}(\mathcal{E})$, is diffeomorphic to

$$
\underset{\varnothing \neq J \subset I}{\#}\left(S^{n-n_{J}-1} \times S^{n_{J}+1}\right)
$$

(C) the trivial open book on $\mathcal{E}, \mathcal{T O} \mathcal{B}(\mathcal{E})$, is diffeomorphic to

$$
\underset{\varnothing \neq J \subset I}{\#}\left(S^{n-n_{J}-1} \times S^{n_{J}+2}\right) .
$$

Proof If $n_{i}>0$ for $i=1, \ldots, k$ then (A) follows from Theorem A1.1 since the hypothesis $q+k \geq 4$ implies that $\mathcal{P}$ has codimension at least 3 in $S^{n}$ and so $\mathcal{E} \times D^{1}$ is simply connected with simply connected boundary. When some $n_{i}$ are zero it follows by induction on the number of them: if we add a new factor $S^{0}$ to a product $\mathcal{P}$ we get a new complement $\mathcal{E}^{\prime}$ which is clearly the connected sum of two copies of $\mathcal{E}$. By Lemma 3 of Section $2.1, \mathcal{E}^{\prime} \times D^{1}$ is the connected sum along the boundary of two copies of $\mathcal{E} \times D^{1}$ and one copy of $S^{n-1} \times D^{2}$ and by the induction hypothesis the collection of summands of the form $S^{n-n_{J}-1} \times D^{n_{J}+2}$ coincides with the one claimed for $\mathcal{E}^{\prime} \times D^{1}$ and (A) is proved. (B) follows from (A) by taking its boundary and (C) by taking its product with $D^{1}$ and again the boundary.

A version of part (B) was proved in the PL-category by McGavran [24] for the case where all $n_{i}=1$ (ie, when $\mathcal{P}$ is the $k$-torus) with a possibly more general hypothesis 
on the embedding. It does not seem that analogues of the other parts of the theorem can be deduced easily from his results.

For our purpose (the proof of Theorem 2.2) we need a different version in the torus case, where the standard nature of the embedding is obtained from a codimension condition.

Theorem A2.3 Consider an embedding of the torus $T^{k}$ in $S^{n}$ with $2 k<n$ and $\mathcal{E}$ the complement of an open tubular neighborhood of $T^{k}$. Then

(A) $\mathcal{E} \times D^{1}$ is diffeomorphic to

$$
\coprod_{j=1}^{k}\left(\begin{array}{l}
k \\
j
\end{array}\right)\left(S^{n-j-1} \times D^{j+2}\right)
$$

(B) the double of $\mathcal{E}, \mathcal{D}(\mathcal{E})$, is diffeomorphic to

$$
\#_{j=1}^{k}\left(\begin{array}{l}
k \\
j
\end{array}\right)\left(S^{n-j-1} \times S^{j+1}\right)
$$

(C) the trivial open book on $\mathcal{E}, \mathcal{T O} \mathcal{B}(\mathcal{E})$, is diffeomorphic to

$$
\#_{j=1}^{k}\left(\begin{array}{l}
k \\
j
\end{array}\right)\left(S^{n-j-1} \times S^{j+2}\right) \text {. }
$$

Proof This follows from the previous theorem since, by [19], any embedding of the torus $T^{k}$ in $S^{n}$ with $2 k<n$ is isotopic to the product considered there.

Obviously a similar result holds for any embedding of the above product of spheres if $q+k-1>\sum_{i=1}^{k} n_{i}$, with refinements depending on the minimum of the $n_{i}$.

\section{References}

[1] D Allen, J La Luz, A counterexample to a conjecture of Bosio and Meersseman, from: "Toric topology", (M Harada, Y Karshon, M Masuda, T Panov, editors), Contemp. Math. 460, Amer. Math. Soc. (2008) 37-45 MR2428347

[2] A Bahri, M Bendersky, F R Cohen, S Gitler, The polyhedral product functor: a method of decomposition for moment-angle complexes, arrangements and related spaces, Adv. Math. 225 (2010) 1634-1668 MR2673742

[3] A Bahri, M Bendersky, $\mathbf{F}$ R Cohen, S Gitler, Cup-products for the polyhedral product functor, Math. Proc. Cambridge Philos. Soc. 153 (2012) 457-469 MR2990625

[4] A Bahri, M Bendersky, F R Cohen, S Gitler, Operations on polyhedral products and a new topological construction of infinite families of toric manifolds (2012) arXiv: $1011.0094 \mathrm{v} 4$ 
[5] I V Baskakov, Cohomology of $K$-powers of spaces and the combinatorics of simplicial divisions, Uspekhi Mat. Nauk 57 (2002) 147-148 MR1992088

[6] I V Baskakov, Triple Massey products in the cohomology of moment-angle complexes, Uspekhi Mat. Nauk 58 (2003) 199-200 MR2035723 In Russian; translated in Russian Math. Surveys 58:5 (2003) 1039-1041

[7] F Bosio, Variétés complexes compactes: une généralisation de la construction de Meersseman et López de Medrano-Verjovsky, Ann. Inst. Fourier (Grenoble) 51 (2001) 1259-1297 MR1860666

[8] F Bosio, L Meersseman, Real quadrics in $\mathbb{C}^{n}$, complex manifolds and convex polytopes, Acta Math. 197 (2006) 53-127 MR2285318

[9] W Browder, Surgery on simply-connected manifolds, Ergeb. Math. Grenzgeb. 65, Springer, New York (1972) MR0358813

[10] V M Buchstaber, T E Panov, Torus actions and their applications in topology and combinatorics, University Lecture Series 24, Amer. Math. Soc. (2002) MR1897064

[11] C Camacho, N H Kuiper, J Palis, The topology of holomorphic flows with singularity, Inst. Hautes Études Sci. Publ. Math. (1978) 5-38 MR516913

[12] M Chaperon, Géométrie différentielle et singularités de systèmes dynamiques, Astérisque 138-139, Société Mathématique de France, Paris (1986) MR858911

[13] M Chaperon, S López De Medrano, Birth of attracting compact invariant submanifolds diffeomorphic to moment-angle manifolds in generic families of dynamics, $\mathrm{C}$. R. Math. Acad. Sci. Paris 346 (2008) 1099-1102 MR2462056

[14] M W Davis, T Januszkiewicz, Convex polytopes, Coxeter orbifolds and torus actions, Duke Math. J. 62 (1991) 417-451 MR1104531

[15] G Denham, A I Suciu, Moment-angle complexes, monomial ideals and Massey products, Pure Appl. Math. Q. 3 (2007) 25-60 MR2330154 Special Issue: In honor of Robert D. MacPherson, Part 3

[16] V Gasharov, I Peeva, V Welker, Coordinate subspace arrangements and monomial ideals, from: "Computational commutative algebra and combinatorics", (T Hibi, editor), Adv. Stud. Pure Math. 33, Math. Soc. Japan, Tokyo (2002) 65-74 MR1890096

[17] F González Acuña, Open Books, Lecture Notes, University of Iowa

[18] B Grünbaum, Convex polytopes, 2nd edition, Graduate Texts in Mathematics 221, Springer, New York (2003) MR1976856

[19] A Haefliger, Differentiable imbeddings, Bull. Amer. Math. Soc. 67 (1961) 109-112 MR0123337

[20] F Hirzebruch, private conversation (1986)

[21] M A Kervaire, J W Milnor, Groups of homotopy spheres, I, Ann. of Math. 77 (1963) 504-537 MR0148075 
[22] J J Loeb, M Nicolau, On the complex geometry of a class of non-Kählerian manifolds, Israel J. Math. 110 (1999) 371-379 MR1750427

[23] M de Longueville, The ring structure on the cohomology of coordinate subspace arrangements, Math. Z. 233 (2000) 553-577 MR1750937

[24] D McGavran, Adjacent connected sums and torus actions, Trans. Amer. Math. Soc. 251 (1979) 235-254 MR531977

[25] S López de Medrano, The space of Siegel leaves of a holomorphic vector field, from: "Holomorphic dynamics", (X Gómez-Mont, J Seade, A Verjovsky, editors), Lecture Notes in Math. 1345, Springer, Berlin (1988) 233-245 MR980961

[26] S López de Medrano, Topology of the intersection of quadrics in $\mathbb{R}^{n}$, from: "Algebraic topology”, (G Carlsson, R L Cohen, H R Miller, D C Ravenel, editors), Lecture Notes in Math. 1370, Springer, Berlin (1989) 280-292 MR1000384

[27] S López de Medrano, A Verjovsky, A new family of complex, compact, nonsymplectic manifolds, Bol. Soc. Brasil. Mat. 28 (1997) 253-269 MR1479504

[28] L Meersseman, A new geometric construction of compact complex manifolds in any dimension, Math. Ann. 317 (2000) 79-115 MR1760670

[29] L Meersseman, A Verjovsky, Holomorphic principal bundles over projective toric varieties, J. Reine Angew. Math. 572 (2004) 57-96 MR2076120

[30] L Meersseman, A Verjovsky, Sur les variétés $L V-M$, from: “Singularities II”, (J-P Brasselet, J L Cisneros-Molina, D Massey, J Seade, B Teissier, editors), Contemp. Math. 475, Amer. Math. Soc. (2008) 111-134 MR2454363

[31] C T C Wall, Classification of $(n-1)$-connected 2n-manifolds, Ann. of Math. 75 (1962) 163-189 MR0145540

[32] C T C Wall, Classification problems in differential topology VI, Classification of ( $s-1)-$ connected $(2 s+1)$-manifolds, Topology 6 (1967) 273-296 MR0216510

[33] C T C Wall, Stability, pencils and polytopes, Bull. London Math. Soc. 12 (1980) 401421 MR593960

Department of Mathematics, Cinvestav, El Colegio Nacional

Centro de Investigacion IPN, Apartado Postal 14740, 14620 Mexico, DF, Mexico

Instituto de Matemáticas, Universidad Nacional Autónoma de México

04510 Mexico, Mexico

samuel.gitler@gmail.com, santiago@matem.unam.mx

Proposed: Steve Ferry

Seconded: Simon Donaldson, Leonid Polterovich
Received: 7 June 2012

Revised: 11 December 2012 